\title{
6. GRAIN-SIZE VARIATIONS WITHIN SLOPE FACIES RECOVERED FROM THE CHILE MARGIN TRIPLE JUNCTION ${ }^{1}$
}

\author{
John A. Diemer ${ }^{2}$ and Randy Forsythe ${ }^{2}$
}

\begin{abstract}
Leg 141 of the Ocean Drilling Program recovered predominantly clays and silty clays from four sites drilled into the inner trench wall and slope in the vicinity of the Chile Margin Triple Junction. Shipboard analysis of the cores indicated significant variations in the physical and chemical properties but limited variation of grain size and mineralogy. Quantitative grain-size analysis has now been completed for a suite of nearly 600 samples obtained directly from the shipboard physical-properties sampling intervals. The analyses provide insights into facies within the finer-grained units deposited on the Chile margin slope as well as into the origin of the variability of the physical properties. The distinctive patterns of sorting and mean grain-size parameters found at each site suggest differential mixing of fine-grained facies. These clay and silty clay facies may include contourites, suspension fallout, distal turbidites, and nepheloid suspensates. Comparisons of downhole variations in the grain-size parameters with the porosity data suggest textural factors have partly controlled porosity loss during burial. The model developed for a tectonically imbricated section at Site 860 is consistent with the cyclic pattern evident in the grain-size data set. Differences seen between holes in the porosity loss curves probably are not caused by grain-size variations, but more likely the result of variations in lithologies on the scale of the depositional basin, as influenced by diagenesis and the tectonic setting.
\end{abstract}

\section{INTRODUCTION}

Leg 141 of the Ocean Drilling Program (ODP) recovered predominantly clays and silty clays from four sites drilled into the inner trench wall and slope environments in the vicinity of the Chile Margin Triple Junction (Fig. 1 shows location of Sites 859, 860, 861, and 863 ). Standard shipboard analysis of the clayey samples recorded limited variation in grain size and mineralogy of these units. In contrast, significant variations in the physical and chemical properties were recorded. For example, the porosity-loss data indicate that from Site 859 (at the toe of the continental slope) up slope to Site 860, and farther up slope to Site 861 , there is a systematic trend to higher porosities at a given depth (Fig. 2A). In addition, Figure $2 \mathrm{~A}$ shows that at a given site (e.g., Site 860), there are significant porosity variations with depth below seafloor. These variations cannot easily be linked to the shipboard descriptive sedimentology. Also of interest is the relationship between the porosity-loss curves obtained at the Leg 141 sites and those curves characteristic of many other clayey and sandy sedimentary sequences reported from other basinal settings (Fig. 2B). Leg 141 samples are very compact in comparison to other basinal settings (Fig. 2B). Are these data evidence for overconsolidation followed by later unroofing for the Leg 141 sites? Or are they the product of other sedimentologic, lithologic, or diagenetic controls affecting the sampled interval? In order to constrain scenarios for overcompaction of the Leg 141 samples, the mineralogic and textural variation of these sediments have been examined using X-ray diffraction (XRD) analysis (see Kurnosov et al., this volume) and postcruise grain-size analysis (this study).

\section{PROCEDURES}

The samples investigated in this study are splits of the shipboard physical-properties samples. Of the 639 physical-properties samples measured during Leg 141, splits of 571 are in the collection at the University of North Carolina at Charlotte (UNCC) (see Table 1). The UNCC samples come from all levels of all cores. Most of the samples

\footnotetext{
'Lewis, S.D., Behrmann, J.H., Musgrave, R.J., and Cande, S.C. (Eds.), 1995. Proc. $O D P$, Sci. Results, 141: College Station, TX (Ocean Drilling Program).

${ }^{2}$ Department of Geography and Earth Sciences, University of North Carolina at Charlotte, Charlotte, NC 28223, U.S.A.
}

are clays and silty clays although some samples contain very fine sand. The grain-size distributions of all 571 samples were measured using a Spectrex ILI-1000 laser particle counter. In addition, 129 of these samples contained significant sand-size material and these sandy samples were processed to extract the fraction coarser than 4 phi. The grain-size distributions of the sand fractions were analyzed in a rapid sediment analyzer (RSA) and the results of the RSA analysis are combined with the laser particle counter data in Table 1.

\section{Laser Particle Counter}

The laser particle counter focuses a laser beam on a suspensate prepared from filtered distilled water and a small amount of the sample. The diffraction of the beam as it strikes particles that cross its path is measured and converted to grain-size data. The instrument is calibrated daily using standards containing particles of known size.

Samples analyzed using the laser particle counter were prepared by extracting $10 \mathrm{mg}$ from the sample bags and gently disaggregating them in a mortar using finger pressure and, when necessary, a pestle. The powdered samples then were placed in capped sample bottles with $10 \mathrm{~mL}$ of distilled water. The bottles were labelled and stored in a dark cabinet to prevent the growth of algae. After a few days, the bottles were placed in an ultrasonic bath for a minimum of five minutes to completely disaggregate the samples. The particles exhibited Brownian motion when a drop of the suspensate was viewed under a petrographic microscope at high power. This indicated that the particles in the suspensates were deflocculated in distilled water (Galehouse, 1971). Dispersants were not used because they contain particles that could alter grain-size distribution data. The sample bottles were stored in a dark cabinet until the final preparation.

For the final preparation, $1 \mathrm{~mL}$ of suspensate was extracted from a thoroughly mixed sample bottle using a pipette and placed into a beaker containing $100 \mathrm{~mL}$ of filtered, distilled water (either 0.2 - or $0.45-\mu \mathrm{m}$ filters were used to filter water throughout the analyses to ensure that the water was free of particles coarser than $1 \mu \mathrm{m})$. From this beaker, $1 \mathrm{~mL}$ of dilute suspensate was extracted using a second pipette and placed into a second beaker of $100 \mathrm{~mL}$ of filtered, distilled water. Prior to placing the sample into the second beaker, a background test was run in order to verify that the $100 \mathrm{~mL}$ of filtered water in the second beaker was indeed free of particles coarser than $1 \mu \mathrm{m}$. Once the sample was placed in the second beaker, a magnetic stir bar 


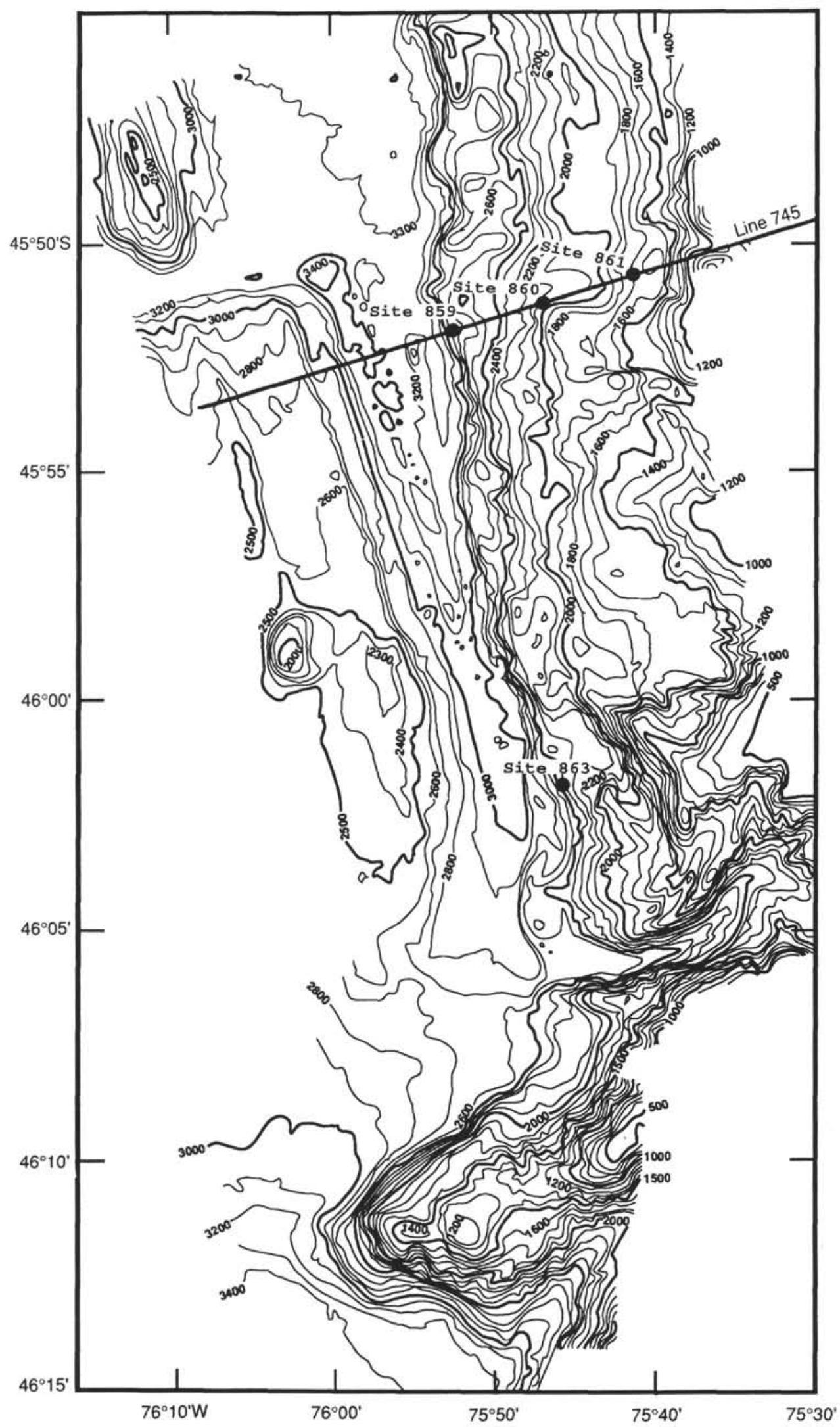

Figure 1. Bathymetric location map for Leg 141 Sites 859, 860, 861, and 863 in the Chile Margin Triple Junction region. Depths are in $100 \mathrm{~m}$ contours. 
A

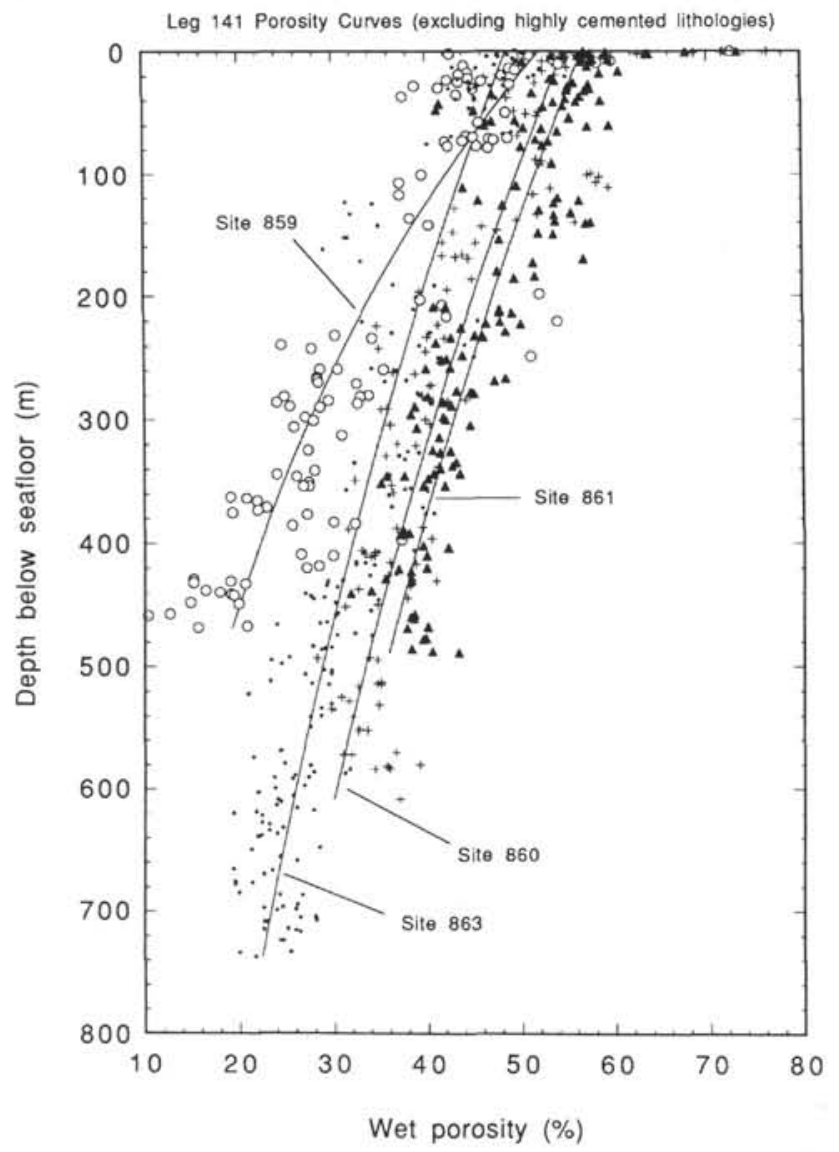

B

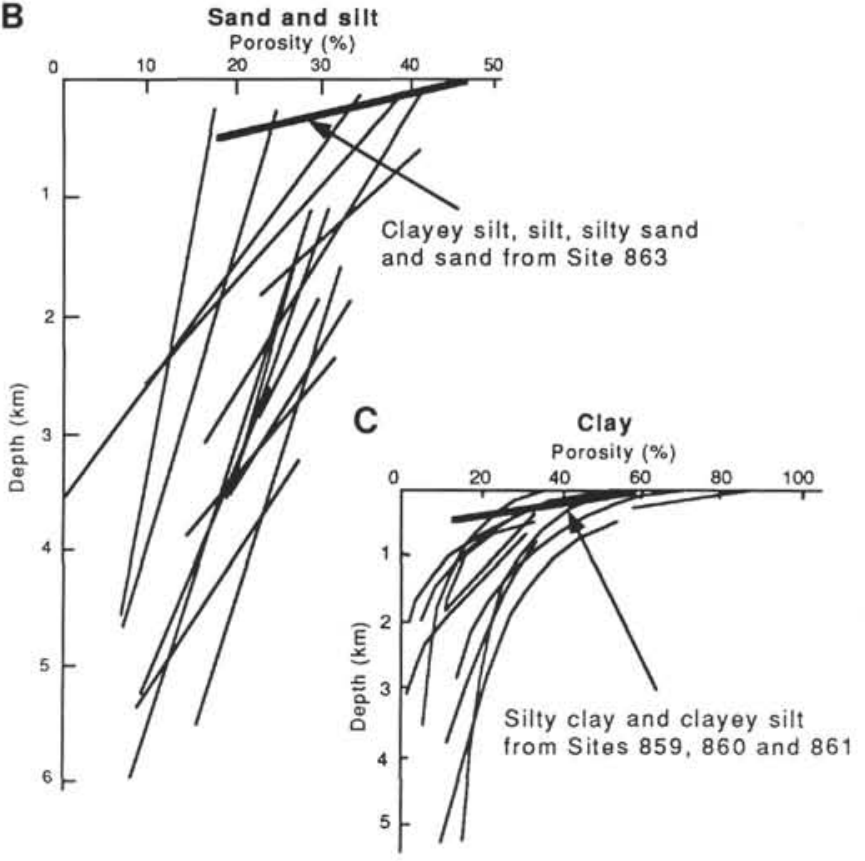

Figure 2. A. Wet porosity plotted as a function of depth below seafloor for Sites $859,860,861$, and 863 . Highly cemented lithologies were excluded from the data in these plots. B. Comparison of porosity-loss curves for Leg 141 sites with data from other sandy sedimentary sequences (modified from Figure 8.15 of Selley, 1988). C. Comparison of porosity-loss curves for Leg 141 sites with data from other clayey sedimentary sequences (modified from Figure 8.7 of Selley, 1988 ).

was added to the beaker. The stir bar was run at a consistent speed sufficient to suspend all the particles present in the beaker so that they all had the opportunity to pass through the laser beam.

The analysis procedure is controlled by the software package supplied by Spectrex (Supercount 6.7). Integrated measurements were made for each sample covering the range of 1 to $100 \mu \mathrm{m}$. Each sample was automatically measured three times and the reported results are the average values from the three runs minus the background signal for the distilled water alone. Thus, by means of the double dilution technique, a representative sample of each physical-properties sample was obtained and measured. Replicate runs of individual samples produced consistent results.

The grain-size analysis data for all 571 samples appear in Table 1. The first column gives the sample reference number. The second column is the depth in meters below seafloor (mbsf) from whence the sample was taken. The third column is the wet porosity of each sample determined by shipboard physical-properties analysis. The fourth, fifth and sixth columns are mean grain size, sorting, and skewness for the grain-size distributions (see formulae in Folk, 1974; Leeder, 1982; McManus, 1988).

The data listed in Table 1 are the product of two analytical techniques. The data for 442 non-sandy samples were generated solely by the laser particle counter. The remaining 129 samples in Table 1 are mixtures of sand and mud and were measured by both the laser particle counter and the rapid sediment analyzer. The results of the two analytical techniques were combined for the 129 sandy samples and can be recognized by the superscript symbol in Table 1 .
The data for the 442 non-sandy samples (measured only by the laser particle counter) are judged to be internally consistent and an accurate representation of grain-size distributions with the possible exception of under-representation of the sand-size fraction. The laser particle counter is designed to measure particles between 1 and 100 micron in diameter. However, the instrument detected little material in the 64 to $100 \mu \mathrm{m}$ range (very fine sand size), even for those samples that, based on visual inspection and sieving, contained very fine sand-size material (coarser than $64 \mu \mathrm{m}$ or 4 phi). It may be that the magnetic stir bar does not suspend the coarser particles within the beaker to the elevation of the laser beam. Alternatively, coarser particles may pass through the beam more rapidly than fine particles and therefore appear as finer particles in the output. As a consequence, it was deemed useful to measure the grain-size distributions of the sand-size fractions separately using the RSA. Regardless of the cause of the possible underrepresentation of the sand-size fractions in the laser particle counter analysis, all samples were prepared and measured using the same procedures. Therefore, variations in grain size for the silt and clay size material as reported by the laser particle counter are considered to be internally consistent and indicative of relative variation in grain size of these fine-grained samples. We used the laser particle counter data from the fine-grained samples to construct Figures 3 through 7.

\section{Rapid Sediment Analyzer}

The rapid sediment analyzer was constructed at UNCC based on a design developed by the Maryland Geological Survey and modified 
Table 1. Textural and porosity data for Leg 141 physical properties residues.

\begin{tabular}{|c|c|c|c|c|c|}
\hline $\begin{array}{l}\text { Core. } \\
\text { section }\end{array}$ & $\begin{array}{l}\text { Depth } \\
\text { (mbst) }\end{array}$ & $\begin{array}{c}\text { Porosity } \\
(\%)\end{array}$ & $\begin{array}{l}\text { Mean } \\
\text { (phi) }\end{array}$ & Sorting & Skewness \\
\hline \multicolumn{6}{|l|}{$141-859 \mathrm{~A}-$} \\
\hline IH-I & 0.52 & 72.6 & 9.27 & 0.47 & -0.08 \\
\hline $2 \mathrm{H}-\mathrm{I}$ & 2.19 & 42.7 & 9.24 & 0.47 & -0.01 \\
\hline $2 \mathrm{H}-2$ & 3.69 & 51.2 & 8.98 & 0.43 & 0.45 \\
\hline $2 \mathrm{H}-3$ & 5.20 & 54.9 & 9.27 & 0.47 & -0.07 \\
\hline $2 \mathrm{H}-4$ & 6.70 & 5.7 & 8.80 & 0.30 & 0.31 \\
\hline $2 \mathrm{H}-5$ & 8.19 & 59.9 & 9.24 & 0.47 & -0.01 \\
\hline $2 \mathrm{H}-6$ & 9.69 & 58.4 & 9.30 & 0.46 & -0.11 \\
\hline $2 \mathrm{H}-7$ & 10.49 & 54.4 & 9.28 & 0.47 & -0.10 \\
\hline $3 \mathrm{H}-\mathrm{I}$ & 11.99 & 44.2 & 9.17 & 0.48 & 0.11 \\
\hline $3 \mathrm{H}-2$ & 13.49 & 50.0 & 9,31 & 0.45 & -0.11 \\
\hline $3 \mathrm{H}-3$ & 13.99 & 49.1 & 9.16 & 0.48 & 0.15 \\
\hline $3 \mathrm{H}-4$ & 15.4 .3 & 49.7 & 9.26 & 0.46 & -0.05 \\
\hline $4 \mathrm{H}-1$ & 17.63 & 44.6 & 9.24 & 0.48 & -0.03 \\
\hline $4 \mathrm{H}-2$ & 19.20 & 43.7 & 9.31 & 0.46 & -0.12 \\
\hline $4 \mathrm{H}-3$ & 19.75 & $48 . .3$ & 9.24 & 0.47 & -0.03 \\
\hline $4 \mathrm{H}-4$ & 22.04 & 44.7 & 9.15 & 0.47 & 0.17 \\
\hline $4 \mathrm{H}-5$ & 2.3 .54 & 42.4 & 9.17 & 0.52 & -0.01 \\
\hline $4 \mathrm{H}-6$ & 24.25 & 46.1 & 9.29 & 0.47 & -0.10 \\
\hline $4 \mathrm{H} \cdot \mathrm{CC}$ & 24.97 & 43.6 & 9.10 & 0.50 & 0.21 \\
\hline $5 \mathrm{H}-2$ & 27.00 & 49.1 & 9.18 & 0.55 & -0.08 \\
\hline $5 \mathrm{H}-4$ & 28.54 & 38.9 & 9.12 & 0.55 & 0.06 \\
\hline $5 \mathrm{H}-5$ & 30.13 & 41.5 & 9.24 & 0.47 & -0.03 \\
\hline $5 \mathrm{H}-6$ & 30.66 & 45.2 & 8.96 & 0.41 & 0.44 \\
\hline $6 x-1$ & 35.70 & 43.4 & 9.05 & 0.46 & 0.39 \\
\hline 6X-CC & 37.17 & 37.6 & 8.88 & 0.36 & 0.42 \\
\hline $8 X-1$ & 50.30 & 48.6 & 8.75 & 0.20 & 0.06 \\
\hline 9P-CC & 58.00 & 45.7 & 8.97 & 0.42 & 0.45 \\
\hline $11 X-1$ & 69.12 & 44,4 & 8.94 & 0.39 & 0.45 \\
\hline $11 X-2$ & 69.90 & 45.1 & 8.76 & 0.17 & -0.02 \\
\hline $11 X-3$ & 71.40 & 46.8 & 9.04 & 0.45 & 0.40 \\
\hline $11 X-3$ & 72.12 & 47.3 & 8.77 & 0.29 & 0.19 \\
\hline $11 X-4$ & 72.90 & 44.0 & 8.78 & 0.27 & 0.24 \\
\hline $11 X-4$ & 73,59 & 42.1 & 9.07 & 0.46 & 0.36 \\
\hline $13 X-1$ & 78.80 & 46.7 & 9.24 & 0.47 & 0.00 \\
\hline $14 X-4$ & 91.86 & & 9.04 & 0.45 & 0.40 \\
\hline $16 \mathrm{X}-\mathrm{CC}$ & 100.70 & 39.6 & 9.03 & 0.46 & 0.39 \\
\hline $16 \mathrm{X}-\mathrm{CC}$ & 100.80 & 38.5 & 9.09 & 0.50 & 0.24 \\
\hline $17 \mathrm{X}-\mathrm{CC}$ & 107.20 & 37.2 & 9.05 & 0.46 & 0.38 \\
\hline 20X-CC & 136.40 & 38.3 & 9.28 & 0.47 & -0.08 \\
\hline \multicolumn{6}{|l|}{$\begin{array}{c}141-859 \mathrm{~B}- \\
4 \mathrm{R}-2\end{array}$} \\
\hline $4 R-2$ & 142.20 & 40.3 & 9.06 & 0.46 & 0.38 \\
\hline 10R-2 & 198.50 & 52.1 & 9.22 & 0.47 & 0.03 \\
\hline 10R-5 & 203.00 & 39.4 & 8.96 & 0.42 & 0.44 \\
\hline IIR-I & 207.30 & 41.7 & 9.08 & 0.47 & 0.35 \\
\hline $12 R-1$ & 216.50 & 42.1 & 9.29 & 0.47 & -0.11 \\
\hline $12 R \cdot 4$ & 220.40 & 53.9 & 9.28 & 0.47 & -0.08 \\
\hline $13 R-6$ & 231.80 & 30.2 & 9.03 & 0.45 & 0.42 \\
\hline I3R-8 & 234.20 & 34.2 & 9.00 & 0.44 & 0.44 \\
\hline $14 R-3$ & 239.30 & 24.5 & 9.26 & 0.46 & -0.03 \\
\hline $14 R-5$ & 242.30 & 27.7 & 9.32 & 0.45 & -0.12 \\
\hline $15 R-3$ & 249.00 & 51.1 & 9.24 & 0.48 & -0.04 \\
\hline $16 \mathrm{R}-3$ & 259.00 & 28.6 & 9.15 & 0.55 & -0.04 \\
\hline $16 \mathrm{R}-4$ & 259.10 & 30.5 & 8.95 & 0.76 & -0.19 \\
\hline $16 R-4$ & 259.60 & 35.4 & 9.19 & 0.60 & -0.19 \\
\hline $17 \mathrm{R}-1$ & 265.60 & 28.3 & 8.90 & 0.75 & -0.12 \\
\hline $17 R-4$ & 269.80 & 28.4 & 9.26 & 0.52 & -0.17 \\
\hline 19R-1 & 284.60 & 29.5 & 8.87 & 0.41 & 0.27 \\
\hline $19 \mathrm{R}-3$ & 287.00 & 32.6 & 9.28 & 0.48 & -0.11 \\
\hline $19 R-4$ & 289.10 & 25.4 & 9.16 & 0.48 & 0.10 \\
\hline $19 R-5$ & 289.90 & 28.6 & 9.19 & 0.49 & 0.05 \\
\hline $20 \mathrm{R}-4$ & 297.90 & 27.0 & 9.19 & 0.48 & 0.04 \\
\hline 20R-6 & 300.70 & 27.9 & 9.05 & 0.46 & 0.39 \\
\hline $2 \mid R-3$ & 306.00 & 25.8 & 9.21 & 0.48 & 0.02 \\
\hline 22R-1 & 312.70 & 30.9 & 8.96 & 0.42 & 0.44 \\
\hline $25 R-1$ & 341.30 & 28.0 & 9.01 & 0.47 & 0.34 \\
\hline $25 R-2$ & 344.20 & 24.0 & 9.11 & 0.53 & 0.12 \\
\hline $25 R-4$ & 346.10 & 26.1 & 9.19 & 0.48 & 0.06 \\
\hline 26R-1 & 351.00 & 27.4 & 9.10 & 0.48 & 0.27 \\
\hline $26 \mathrm{R}-3$ & 354.00 & 26.8 & 9.22 & 0.47 & 0.02 \\
\hline $27 R-2$ & 362.70 & 19.1 & 9.16 & 0.50 & 0.07 \\
\hline $27 R-3$ & 363.80 & 20.8 & 9.19 & 0.54 & -0.08 \\
\hline $27 R-4$ & 365.90 & 21.9 & 9.24 & 0.52 & -0.14 \\
\hline $28 \mathrm{R}-1$ & 371.20 & 22.9 & 9.13 & 0.52 & 0.09 \\
\hline $28 R-3$ & 373,40 & 22.0 & 9.16 & 0.56 & -0.06 \\
\hline $28 R-4$ & 375.40 & 19.3 & 8.99 & 0.51 & 0.26 \\
\hline 28R-CC & 376.90 & 27.2 & 9.09 & 0.51 & 0.21 \\
\hline $29 R-3$ & 383.20 & 30.0 & 8.84 & 0.32 & 0.39 \\
\hline $29 \mathrm{R}-4$ & 384.20 & 32.3 & 8.76 & 0.19 & 0.08 \\
\hline $29 \mathrm{R}-5$ & 385.80 & 25.6 & 8.73 & 0.23 & -0.19 \\
\hline 30R-CC & 397.40 & 37.2 & 8.75 & 0.32 & 0.06 \\
\hline $32 \mathrm{R}-1$ & 409.00 & 26.5 & 8.95 & 0.43 & 0.40 \\
\hline $32 \mathrm{R}-2$ & 410.10 & 29.9 & 8.82 & 0.33 & 0.29 \\
\hline $33 R-1$ & 418.40 & 28.4 & 8.81 & 0,30 & 0.33 \\
\hline 33R-2 & 420.10 & 27.1 & 8.75 & 0.35 & 0.08 \\
\hline $34 \mathrm{R}-1$ & 429.20 & 15.1 & 8.74 & 0.19 & -0.10 \\
\hline
\end{tabular}

\begin{tabular}{|c|c|c|c|c|c|}
\hline $\begin{array}{l}\text { Core, } \\
\text { section }\end{array}$ & $\begin{array}{l}\text { Depth } \\
\text { (mbsf) }\end{array}$ & $\begin{array}{c}\text { Porosity } \\
(\%)\end{array}$ & $\begin{array}{l}\text { Mean } \\
\text { (phi) }\end{array}$ & Sorting & Skewness \\
\hline $34 \mathrm{R}-2$ & 430.70 & 19.0 & 8.74 & 0.16 & 0.00 \\
\hline $34 \mathrm{R}-3$ & 431.90 & 15.1 & 8.76 & 0.25 & 0.12 \\
\hline $34 \mathrm{R}-4$ & 432.60 & 20.6 & 9.06 & 0.46 & 0.39 \\
\hline $35 R-1$ & 438.20 & 16.4 & 9.13 & 0.47 & 0.24 \\
\hline $35 \mathrm{R}-2$ & 4.39 .70 & 17.9 & 9.10 & 0.47 & 0.31 \\
\hline $35 R-3$ & 441.20 & 19.1 & 9.18 & 0.51 & 0.01 \\
\hline $35 R-4$ & 442.20 & 19.4 & 9.15 & 0.54 & 0.00 \\
\hline $36 \mathrm{R}-1$ & 448.00 & 14.8 & 9.20 & 0.49 & 0.00 \\
\hline $36 \mathrm{R}-2$ & 448.90 & 19.9 & 9.05 & 0.52 & 0.24 \\
\hline $37 R-1$ & 457.10 & 12.6 & 9.18 & 0.48 & 0.06 \\
\hline $37 \mathrm{R}-2$ & 458.60 & 10.3 & 8.71 & 0.26 & -0.24 \\
\hline $38 \mathrm{R}-1$ & 467.10 & 20.8 & 8.73 & 0.21 & -0.20 \\
\hline \multicolumn{6}{|l|}{$141-860 \mathrm{~A}-$} \\
\hline $\mathrm{IH}-2$ & 1.80 & 68.7 & 9.04 & 0.52 & 0.23 \\
\hline $1 \mathrm{H}-2$ & 2.60 & 62.8 & 8.82 & 0.35 & 0.25 \\
\hline $\mathrm{IH}-4$ & 5.60 & 58.2 & 8.92 & 0.44 & 0.32 \\
\hline $1 \mathrm{H}-3$ & 4.10 & 56.3 & 8.85 & 0.4 & 0.25 \\
\hline $1 \mathrm{H}-7$ & 9.29 & 57.5 & 8.73 & 0.27 & 0.00 \\
\hline \multicolumn{6}{|l|}{$141-860 \mathrm{~B}-$} \\
\hline $1 \mathrm{H}-1$ & 1.00 & 71.6 & 8.73 & 0.29 & 0.00 \\
\hline $2 \mathrm{H}-2$ & 4.05 & 53.5 & 8.83 & 0.47 & 0.13 \\
\hline $2 \mathrm{H}-3$ & 5.51 & 58.8 & 8.87 & 0.39 & 0.29 \\
\hline $2 \mathrm{H}-4$ & 6.49 & 58.9 & 8.93 & 0.51 & 0.26 \\
\hline $2 \mathrm{H}-5$ & 8.36 & 53.9 & 9.08 & 0.51 & 0.22 \\
\hline $2 \mathrm{H}-6$ & 9.88 & 53.7 & 8.67 & 0,34 & -0.20 \\
\hline $3 \mathrm{H}-2$ & 13,40 & 55.2 & 8.88 & 0.47 & 0.24 \\
\hline $3 \mathrm{H}-4$ & 16.40 & 52.6 & 9.05 & 0.46 & 0.39 \\
\hline $3 \mathrm{H}-6$ & 19.40 & 54.0 & 8.73 & 0.48 & $-0.30 \$$ \\
\hline $3 \mathrm{H}-6$ & 19.10 & 47.9 & 8.65 & 1.25 & $-0.60 \$$ \\
\hline $4 \mathrm{H}-1$ & 21.40 & 46.4 & 9.03 & 0.52 & 0.23 \\
\hline $4 \mathrm{H}-4$ & 25.90 & 51.8 & 8.99 & 0.53 & 0.21 \\
\hline $4 \mathrm{H}-6$ & 28.90 & 45.5 & 8.97 & 0.6 & 0.09 \\
\hline $5 \mathrm{H}-2$ & 32.40 & 49.0 & 9.01 & 0.52 & 0.23 \\
\hline $5 \mathrm{H}-5$ & 36.80 & 47.2 & 8.77 & 0.51 & 0.06 \\
\hline $6 \mathrm{H}-3$ & 42.59 & & 3.11 & 1.10 & $0.45 \$$ \\
\hline $6 \mathrm{H}-3$ & 43.40 & & 3.16 & 1.21 & $0.49 \S$ \\
\hline $6 \mathrm{H}-6$ & 47.14 & & 3.13 & 1.13 & $0.45 \$$ \\
\hline $7 \mathrm{H}-1$ & 49.17 & 49.5 & 8.67 & 0.33 & -0.40 \\
\hline $7 \mathrm{H}-1$ & 49.84 & 52.1 & 8.68 & 0.27 & -0.30 \\
\hline $7 \mathrm{H}-2$ & 51.22 & 50.8 & 8.75 & 0.4 & 0.00 \\
\hline $7 \mathrm{H}-3$ & 53.00 & 51.9 & 8.82 & 0.39 & 0.19 \\
\hline $12 X-1$ & 88.32 & 51.8 & 8.77 & 0.39 & 0.04 \\
\hline $12 X-2$ & 89.85 & 52.4 & 8.71 & 0.25 & -0.20 \\
\hline $12 X-3$ & 91.13 & 53.4 & 8.59 & 0.36 & -0.40 \\
\hline $12 X-4$ & 92.81 & 52.2 & 8.71 & 0.2 .3 & -0.20 \\
\hline $14 x-1$ & 99.57 & 57.7 & 8.74 & 0.21 & -0.20 \\
\hline $14 X-2$ & 101.00 & 57.3 & 8.73 & 0.21 & -0.20 \\
\hline $14 X-C C$ & 102.30 & 58.5 & 8.68 & 0.27 & -0.30 \\
\hline $15 X-1$ & 107.30 & 58.2 & 8.88 & 0.35 & 0.42 \\
\hline $15 X-3$ & 111.10 & 59.5 & 8.75 & 0.39 & 0.01 \\
\hline $15 \times-4$ & 112.20 & 53.3 & 8.75 & 0.39 & 0.00 \\
\hline $16 X-1$ & 117.10 & 51.5 & 8.60 & 0.49 & -0.30 \\
\hline $16 \mathrm{X}-\mathrm{CC}$ & 120.40 & 49.5 & 8.50 & 0.47 & -0.50 \\
\hline $17 X-2$ & 128.30 & 4.3 .1 & 8.79 & 0.65 & -0.10 \\
\hline $17 \mathrm{X} \cdot \mathrm{CC}$ & 133.70 & 51.9 & 8.96 & 0.49 & 0.28 \\
\hline $19 \times-1$ & 138.40 & 49.7 & 8.92 & 0.48 & 0.25 \\
\hline $19 \times-2$ & 139.90 & 55.9 & 8.55 & 0.45 & -0.40 \\
\hline $19 \times-5$ & 143.50 & 46.0 & 8.44 & 1.25 & $-0.65 \$$ \\
\hline $20 \mathrm{X} \cdot \mathrm{CC}$ & 148.00 & 42.9 & 8.63 & 0.55 & -0.30 \\
\hline $21 X-1$ & 156.40 & 41.8 & 8.47 & 0.68 & -0.30 \\
\hline $21 \mathrm{X}-\mathrm{CC}$ & 156.90 & 45.3 & 8.86 & 0.48 & 0.18 \\
\hline $22 X-1$ & 166.30 & 43.9 & 8.86 & 0.64 & 0.00 \\
\hline $22 X-2$ & 167.30 & 41.7 & 8.59 & 0.45 & $-0.40 \S$ \\
\hline $25 \mathrm{X}-\mathrm{CC}$ & 196.40 & 39.3 & 8.59 & 0.48 & $-0.40 \S$ \\
\hline $28 \mathrm{X}-1$ & 223.30 & 41.2 & 8.78 & 0.38 & 0.11 \\
\hline $28 \mathrm{X}-\mathrm{CC}$ & 224.50 & 34.7 & 8.71 & 0.43 & -0.10 \\
\hline $29 X-1$ & 233.40 & 40.0 & 8.79 & 0.36 & 0.14 \\
\hline $30 \times-3$ & 244.80 & 39.9 & 8.98 & 0.62 & 0.08 \\
\hline $31 \times-2$ & 253.70 & 41.5 & 8.53 & 0.61 & -0.20 \\
\hline $32 X-1$ & 261.80 & 36.4 & 8.92 & 0.67 & 0.00 \\
\hline $32 X-2$ & 263.20 & 38.7 & 9.00 & 0.58 & 0.13 \\
\hline $33 x-1$ & 272.40 & 40.5 & 8.71 & 0.40 & 0.00 \\
\hline $33 \mathrm{X}-2$ & 272.90 & 40.3 & 8.94 & 0.48 & 0.28 \\
\hline $34 X-1$ & 281.10 & 39.5 & 9.05 & 0.53 & 0.18 \\
\hline $34 \times-2$ & 282.60 & 40.2 & 8.82 & 0.43 & 0.17 \\
\hline $34 X-3$ & 284.10 & 44.1 & 8.69 & 0.35 & -0.10 \\
\hline $34 X-4$ & 285.00 & 40.7 & 8.92 & 0.51 & 0.21 \\
\hline $34 \mathrm{X}-\mathrm{CC}$ & 285.50 & 42.2 & 8.88 & 0.46 & 0.22 \\
\hline $35 X-1$ & 291.20 & 35.8 & 8.78 & 0.52 & 0.04 \\
\hline $35 \mathrm{X}-\mathrm{CC}$ & 292.00 & 35.1 & 9.02 & 0.56 & 0.16 \\
\hline $36 \mathrm{X}-1$ & 300.30 & 39.8 & 9.00 & 0.52 & 0.24 \\
\hline $36 X-3$ & 303.50 & 40.3 & 9.08 & 0.55 & 0.14 \\
\hline $36 \times-4$ & 304.30 & 36.1 & 8.80 & 0.55 & 0.01 \\
\hline $38 \mathrm{X}-1$ & 320.00 & 36.8 & 9.05 & 0.55 & 0.14 \\
\hline $39 \times-1$ & 329.80 & 35.6 & 8.68 & 0.51 & -0.10 \\
\hline
\end{tabular}


Table 1 (continued).

\begin{tabular}{|c|c|c|c|c|c|}
\hline $\begin{array}{l}\text { Core. } \\
\text { section }\end{array}$ & $\begin{array}{l}\text { Depth } \\
\text { (mbsf) }\end{array}$ & $\begin{array}{c}\text { Porosity } \\
(\%)\end{array}$ & $\begin{array}{l}\text { Mean } \\
\text { (phi) }\end{array}$ & Sorting & Skewness \\
\hline $40 \mathrm{X}-\mathrm{CC}$ & 338.50 & 41.2 & 8.53 & 0.49 & -0.50 \\
\hline $41 X-1$ & 349.40 & 32.3 & 8.45 & 0.46 & -0.40 \\
\hline $41 \times-4$ & 353.50 & 36.2 & 8.81 & 0.53 & 0.07 \\
\hline $43 X-1$ & 359.50 & 36.4 & 8.68 & 0.67 & -0.10 \\
\hline $46 X-1$ & 387.20 & 39.5 & 8.75 & 0.64 & -0.10 \\
\hline $46 X-2$ & 388.20 & 36.7 & 8.84 & 0.74 & -0.10 \\
\hline $46 \mathrm{X}-\mathrm{CC}$ & 389.20 & 31.5 & 8.81 & 0.68 & 0.00 \\
\hline $48 X-1$ & 406.30 & 33.0 & 8.45 & 0.79 & -0.20 \\
\hline $48 X-2$ & 407.50 & 34.4 & 9.09 & 0.60 & 0.00 \\
\hline $48 X-3$ & 408.30 & 33.3 & 8.92 & 0.62 & 0.07 \\
\hline $48 \times-4$ & 410.10 & 34.3 & 8.23 & 0.62 & -0.30 \\
\hline $48 X-5$ & 411.40 & 33.9 & 8.81 & 0.66 & 0.00 \\
\hline $49 X-1$ & 415.90 & 36.0 & 8.73 & 0.75 & -0.10 \\
\hline $49 \mathrm{X}-\mathrm{CC}$ & 416.80 & 38.7 & 8.68 & 0.60 & -0.10 \\
\hline $50 \mathrm{X}-1$ & 426.10 & 35.8 & 8.99 & 0.57 & 0.15 \\
\hline $50 \times-4$ & 429.60 & 38.3 & 8.83 & 0.49 & 0.13 \\
\hline $50 \mathrm{X}-\mathrm{CC}$ & 4.30 .80 & 40.9 & 8.76 & 0.51 & 0.01 \\
\hline $51 X-1$ & 435.20 & 38.1 & 8.86 & 0.55 & 0.11 \\
\hline $51 X-2$ & 4.37 .30 & 32.6 & 8.46 & 0.70 & -0.30 \\
\hline $52 \mathrm{X}-\mathrm{CC}$ & 444.60 & 37.8 & 8.68 & 0.65 & -0.10 \\
\hline $53 \mathrm{X}-1$ & 450.00 & 34.7 & 8.60 & 0.64 & -0.20 \\
\hline $53 X-2$ & 451.40 & 31.2 & 8.99 & 0.59 & 0.12 \\
\hline $58 X-1$ & 492.80 & 33.8 & 8.40 & 0.78 & $-0.44 \$$ \\
\hline $58 \mathrm{X}-1$ & 493.50 & 28.2 & 8,64 & 0.69 & -0.10 \\
\hline $58 X-2$ & 494.80 & 34.7 & 8.85 & 0.67 & 0.01 \\
\hline $60 X-1$ & 512.70 & 35.0 & 9.17 & 0.56 & -0.10 \\
\hline $60 \times-2$ & 514.10 & 34.6 & 8.55 & 0.59 & -0.20 \\
\hline $60 X-3$ & 514.90 & 35.0 & 8.94 & 0.61 & 0.09 \\
\hline $60 X-4$ & 516.40 & 32.6 & 9.00 & 0.54 & 0.21 \\
\hline $61 X-3$ & 525.40 & 30.8 & 8.51 & 0.75 & -0.20 \\
\hline $61 \times-5$ & 528.70 & 31.6 & 8.64 & 0.55 & -0.30 \\
\hline $61 X-C C$ & 531.30 & 34.8 & 9.08 & 0.55 & 0.11 \\
\hline $62 X-1$ & 531.60 & 34.8 & 9.06 & 0.54 & 0.17 \\
\hline $62 X-3$ & 534.90 & 29.7 & 9.02 & 0.51 & 0.26 \\
\hline $64 X-1$ & 551.00 & 32.7 & 8.99 & 0.54 & 0.20 \\
\hline $64 X-2$ & 552.00 & 33.6 & 9.06 & 0.51 & 0.2 .3 \\
\hline $67 X-1$ & 580.40 & 39.1 & 9.10 & 0.55 & 0.08 \\
\hline $67 X-2$ & 581.60 & 35.6 & 8.91 & 0.50 & 0.24 \\
\hline $67 \times-3$ & 583.10 & 35.9 & 9.01 & 0.50 & 0.26 \\
\hline $67 X-4$ & 584.10 & 34.4 & 8.99 & 0.54 & 0.21 \\
\hline $70 \mathrm{x}-\mathrm{CC}$ & 608.40 & 37.0 & 8.99 & 0.51 & 0.26 \\
\hline \multicolumn{6}{|l|}{$141-861 \mathrm{~A}=$} \\
\hline $\begin{array}{l}\mathrm{IH}-1 \\
\mathrm{IH}-1\end{array}$ & 0.69 & 57.0 & 8.97 & 0.50 & 0.26 \\
\hline $\begin{array}{l}\mathrm{IH}-1 \\
1 \mathrm{H}-2\end{array}$ & 1.45 & 59.4 & 8.77 & 0.34 & 0.10 \\
\hline $\begin{array}{l}1 \mathrm{H}-2 \\
1 \mathrm{H}-3\end{array}$ & 2.50 & 57.7 & 8.87 & 0.35 & 0.42 \\
\hline $1 \mathrm{H}-3$ & 3.98 & 54.9 & 8.76 & 0.32 & 0.12 \\
\hline $1 \mathrm{H}-5$ & 7.00 & 58.0 & 8.81 & 0.43 & 0.14 \\
\hline $1 \mathrm{H}-6$ & 8.50 & 56.7 & 8.54 & 0.42 & -0.50 \\
\hline \multicolumn{6}{|l|}{$141-861 \mathrm{~B}-$} \\
\hline $1 \mathrm{H}-1$ & 1.00 & 67.8 & 8.86 & 0.51 & 0.14 \\
\hline IH-2 & 2.50 & 63.6 & 8.97 & 0.54 & 0.21 \\
\hline $\mathrm{IH}-3$ & 4.00 & 56.4 & 8.76 & 0.51 & 0.01 \\
\hline $1 \mathrm{H}-4$ & 5.50 & 56.9 & 8.82 & 0.56 & 0.03 \\
\hline $1 \mathrm{H}-5$ & 7.02 & 59.6 & 8.65 & 0.43 & -0.20 \\
\hline IH-6 & 8.49 & 50.5 & 8.56 & 0.57 & -0.30 \\
\hline \multicolumn{6}{|l|}{$141-861 \mathrm{C}-$} \\
\hline $\mathrm{IH}-1$ & 1.00 & 73.3 & 8.45 & 0.50 & -0.40 \\
\hline $1 \mathrm{H}-2$ & 2.50 & 63.9 & 8.60 & 0.50 & -0.20 \\
\hline $2 \mathrm{H}-1$ & 4.00 & 53.6 & 8.43 & 0.44 & -0.30 \\
\hline $2 \mathrm{H}-3$ & 7.00 & 59.6 & 8.46 & 0.44 & -0.30 \\
\hline $2 \mathrm{H}-4$ & 8.50 & 57.7 & 8.52 & 0.52 & -0.40 \\
\hline $2 \mathrm{H}-7$ & 12.40 & 57.4 & 8.93 & 0.53 & 0.18 \\
\hline $3 \mathrm{H}-3$ & 16.60 & 60.7 & 8.72 & 0.24 & -0.20 \\
\hline $3 \mathrm{H}-4$ & 18.00 & 58.7 & 8.75 & 0.34 & 0.04 \\
\hline $4 \mathrm{H}-1$ & 22.80 & 53.4 & 8.91 & 0.49 & 0.23 \\
\hline $4 \mathrm{H}-2$ & 24.30 & 53.9 & 8.94 & 0.54 & 0.19 \\
\hline $4 \mathrm{H}-3$ & 25.80 & 55.9 & 8.74 & 0.34 & 0.01 \\
\hline $4 \mathrm{H}-4$ & 27.40 & 57.5 & 8.72 & 0.29 & -0.10 \\
\hline $4 \mathrm{H}-5$ & 28.90 & 55.3 & 8.66 & 0.40 & -0.20 \\
\hline $4 \mathrm{H}-6$ & 30.40 & 57.6 & 8.75 & 0.39 & 0.02 \\
\hline $5 \mathrm{H}-2$ & 32.50 & 57.1 & 8.92 & 0.49 & 0.25 \\
\hline $5 \mathrm{H}-3$ & 34.10 & 51.5 & 8.80 & 0.40 & 0.16 \\
\hline $5 \mathrm{H}-4$ & 35.00 & 47.2 & 8.71 & 0.35 & 0.00 \\
\hline $5 \mathrm{H}-5$ & 37.10 & 56.5 & 8.58 & 0.39 & -0.40 \\
\hline $5 \mathrm{H}-6$ & 39.00 & 55.0 & 8.84 & 0.51 & 0.15 \\
\hline $5 \mathrm{H}-7$ & 40.40 & 58.7 & 8.87 & 0.45 & 0.23 \\
\hline $5 \mathrm{H}-8$ & 41.50 & 56.1 & 8.65 & 0.33 & -0.40 \\
\hline $6 \mathrm{H}-1$ & 41.70 & 53.7 & 8.68 & 0.51 & $-0.33 \&$ \\
\hline $6 \mathrm{H}-2$ & 43.20 & 41.5 & 8.84 & 0.55 & 0.10 \\
\hline $6 \mathrm{H}-3$ & 44.70 & 54.7 & 8,99 & 0.50 & 0.27 \\
\hline $6 \mathrm{H}-4$ & 45.70 & 52.6 & 8.23 & 0.59 & -0.30 \\
\hline $6 \mathrm{H}-6$ & 48.60 & 45.2 & 8.72 & 0.63 & -0.10 \\
\hline $6 \mathrm{H}-5$ & 48.00 & 41.2 & 8.43 & 0.53 & -0.40 \\
\hline $7 \mathrm{H}-3$ & 54.50 & 55.4 & 8.53 & 0.49 & -0.40 \\
\hline
\end{tabular}

\begin{tabular}{|c|c|c|c|c|c|}
\hline $\begin{array}{l}\text { Core. } \\
\text { section }\end{array}$ & $\begin{array}{l}\text { Depth } \\
\text { (mbsf) }\end{array}$ & $\begin{array}{c}\text { Porosity } \\
(\%)\end{array}$ & $\begin{array}{l}\text { Mean } \\
\text { (phi) }\end{array}$ & Sorting & Skewness \\
\hline $7 \mathrm{H}-3$ & 54.10 & 55.4 & 8.52 & 1.19 & -0.618 \\
\hline $7 \mathrm{H}-4$ & 56.40 & 49.6 & 6.41 & 2.38 & $-0.61 \S$ \\
\hline $7 \mathrm{H}-5$ & 57.10 & 47.1 & 5.44 & 2.34 & $0.80 \$$ \\
\hline $7 \mathrm{H}-7$ & 60.50 & 59.6 & 8.88 & 0.56 & 0.11 \\
\hline $7 \mathrm{H}-8$ & 61.10 & 57.3 & 8.39 & 0.79 & -0.558 \\
\hline $8 \mathrm{H}-1$ & 60.30 & 46.3 & 5.76 & 3.23 & $-0.8 .3 \$$ \\
\hline $8 \mathrm{H}-2$ & 62.60 & 50.5 & 8.85 & 0.72 & -0.10 \\
\hline $8 \mathrm{H}-3$ & 63.40 & 52.4 & 8.51 & 0.49 & -0.40 \\
\hline $8 \mathrm{H}-4$ & 65.50 & 54.1 & 8.36 & 1.31 & $-0.67 \$$ \\
\hline $10 \mathrm{H}-1$ & 72.10 & 51.8 & 8.40 & 0.56 & $-0.50^{\prime \prime}$ \\
\hline $10 \mathrm{H}-2$ & 73.50 & 53.1 & 8.75 & 0.38 & 0.02 \\
\hline $10 \mathrm{H}-4$ & 76.70 & 52.5 & 9.03 & 0.61 & 0.06 \\
\hline $10 \mathrm{H}-5$ & 77.90 & 50.2 & 8.58 & 0.59 & -0.30 \\
\hline $12 X-2$ & 91.80 & 53.5 & 8.69 & 0.58 & -0.10 \\
\hline $14 X-1$ & 110.00 & 49.7 & 8.76 & 0.61 & -0.10 \\
\hline $14 X-2$ & 111.00 & 44.0 & 8.58 & 0.53 & -0.30 \\
\hline $15 x-1$ & 120.00 & 54.1 & 8.68 & 0.51 & -0.20 \\
\hline $15 X-2$ & 122.00 & 45.6 & 8.69 & 0.42 & -0.298 \\
\hline $15 X-3$ & 122.00 & 56.4 & 8.54 & 0.65 & $-0.53 \$$ \\
\hline $15 \times-4$ & 124.00 & 53.7 & 8.50 & 0.54 & -0.40 \\
\hline $15 X-5$ & 126.00 & 48.2 & 8.75 & 0.55 & 0.00 \\
\hline $16 x-3$ & 132.00 & 55.5 & 8.77 & 0.52 & $-0.32 \$$ \\
\hline $16 X-2$ & 130.00 & 52.1 & 8.42 & 0.38 & $-0.61 \$$ \\
\hline $16 \times-4$ & 134.00 & 5.3 .7 & 8.57 & 0.45 & -0.40 \\
\hline $17 X-1$ & 139.00 & 53.8 & 8.64 & 0.52 & -0.20 \\
\hline $17 x-1$ & 140.00 & 57.6 & 8.75 & 0.34 & $-0.18 \$$ \\
\hline $17 \mathrm{X}-\mathrm{CC}$ & 141.00 & 57.1 & 8.99 & 0.56 & 0.16 \\
\hline $18 X-1$ & 149.00 & 52.0 & 8.97 & 0.64 & 0.04 \\
\hline $18 X-2$ & 150.00 & 53.6 & 8.39 & 0.70 & $-0.55 \$$ \\
\hline $18 X-4$ & 154.00 & 47.8 & 9.10 & 0.51 & 0.18 \\
\hline $21 X-1$ & 170.00 & 56.8 & 9.00 & 0.53 & 0.22 \\
\hline $22 \mathrm{X}-1$ & 180.00 & 47.6 & 8.64 & 0.71 & $-0.47 \$$ \\
\hline $22 \times-5$ & 186.00 & 49.4 & 9.01 & 0.62 & 0.07 \\
\hline $22 X-5$ & 186.00 & 27.2 & 8.83 & 0.61 & 0.05 \\
\hline $22 X-3$ & 184.00 & 51.6 & 8.53 & 0.45 & -0.40 \\
\hline $25 X-1$ & 209.00 & 40.8 & 8.50 & 0.66 & -0.30 \\
\hline $25 X-2$ & 211.00 & 47.8 & 8.85 & 0.55 & 0.10 \\
\hline $25 X-3$ & $21,3.00$ & 47.7 & 8.89 & 0.49 & 0.21 \\
\hline $27 X-1$ & 221.00 & 47.8 & 8.80 & 0.47 & 0.09 \\
\hline $27 X-2$ & 222.00 & 46.3 & 8.66 & 0.45 & -0.20 \\
\hline $27 X-2$ & 222.00 & 46.3 & 8.66 & 0.45 & -0.20 \\
\hline $27 X-3$ & 223.00 & 50.0 & 8.62 & 0.43 & -0.30 \\
\hline $27 X-5$ & 226.00 & 43.7 & 8.72 & 0.27 & -0.20 \\
\hline $28 X-1$ & 229.00 & 48.4 & 8.59 & 0.35 & -0.40 \\
\hline $28 X-3$ & 232.00 & 45.1 & 8.60 & 0.37 & -0.40 \\
\hline $28 X-4$ & $23,3.00$ & 46.0 & 8.41 & 0.53 & -0.50 \\
\hline $28 X-5$ & 234.00 & 42.6 & 8.26 & 0.59 & -0.40 \\
\hline $29 X-1$ & 238.00 & 41.0 & 8.45 & 0.49 & -0.40 \\
\hline $31 X-1$ & 248.00 & 43.8 & 8.62 & 0.54 & -0.30 \\
\hline $31 X-2$ & 251.00 & 42.1 & 8.59 & 0.64 & -0.20 \\
\hline $31 X-3$ & 252.00 & 41.6 & 8.23 & 0.61 & -0.40 \\
\hline $32 X-1$ & 258.00 & 42.5 & 8.62 & 0.57 & -0.30 \\
\hline $32 X-2$ & 259.00 & 39.9 & 8.35 & 0.59 & -0.50 \\
\hline $33 X-1$ & 267.00 & 48.4 & 8.63 & 0.45 & -0.30 \\
\hline $33 X-2$ & 269.00 & 47.2 & 8.68 & 0.44 & $-0.31 \$$ \\
\hline $34 X-2$ & 277.00 & 43.2 & 8.43 & 0.49 & -0.40 \\
\hline $34 X-3$ & 278.00 & 44.7 & 8.25 & 0.61 & -0.40 \\
\hline $34 X-4$ & 279.00 & 45.0 & 8.85 & 0.62 & 0.05 \\
\hline $34 X-6$ & 282.00 & 40.1 & 8.74 & 0.74 & -0.10 \\
\hline $35 X-1$ & 286.00 & 41.7 & 8.53 & 0.60 & -0.20 \\
\hline $35 X-3$ & 289.00 & 42.6 & 8.84 & 0.65 & 0.00 \\
\hline $35 X-4$ & 290.00 & 38.7 & 8.96 & 0.64 & 0.02 \\
\hline $36 \mathrm{X}-1$ & 296.00 & 38.3 & 8.81 & 0.75 & -0.10 \\
\hline $36 \times-2$ & 298.00 & 41.7 & 8.55 & 0.60 & -0.20 \\
\hline $36 \times-4$ & 300.00 & 42.0 & 8.69 & 0.58 & -0.10 \\
\hline $37 X-1$ & 305.00 & 44.6 & 8.62 & 0.53 & -0.20 \\
\hline $37 \times-2$ & 307.00 & 38.9 & 9.01 & 0.55 & 0.18 \\
\hline $38 \mathrm{X}-\mathrm{CC}$ & 315.00 & 41.3 & 8.86 & 0.57 & 0.09 \\
\hline $39 \times-1$ & 325.00 & 40.6 & 8.57 & 0.44 & -0.40 \\
\hline $39 X-2$ & 326.00 & 42.5 & 8.50 & 0.46 & -0.50 \\
\hline $40 \times-1$ & 335.00 & 43.1 & 8.52 & 0.48 & -0.358 \\
\hline $40 x-3$ & 338.00 & 42.7 & 8.35 & 0.67 & -0.648 \\
\hline $40 X-6$ & 340.00 & 41.4 & 9.12 & 0.60 & -0.10 \\
\hline $4 I X-1$ & 344.00 & 40.7 & 8.50 & 0.53 & -0.40 \\
\hline $41 X-2$ & 346.00 & 35.7 & 9.10 & 0.54 & 0.12 \\
\hline $41 X-4$ & 348.00 & 40.1 & 8.95 & 0.54 & 0.18 \\
\hline \multicolumn{6}{|l|}{$141-86 \mid \mathrm{D}$ - } \\
\hline IR-2 & 344.00 & 43,5 & 8.55 & $\begin{array}{l}0.45 \\
0.37\end{array}$ & $\begin{array}{l}-0.30 \\
-0.40\end{array}$ \\
\hline IR-3 & 346.00 & 37.6 & 8.60 & 0.37 & -0.40 \\
\hline $2 \mathrm{R}-1$ & 352.00 & 35.1 & 8.41 & 0.55 & -0.40 \\
\hline $2 \mathrm{R}-2$ & 354.00 & 41.9 & 8.68 & 0.36 & -0.20 \\
\hline $2 \mathrm{R}-2$ & 354.00 & 39.6 & 8.39 & 0.55 & -0.50 \\
\hline $6 \mathrm{R}-1$ & 391.00 & 37.5 & 8.47 & 0.51 & -0.50 \\
\hline $6 \mathrm{R}-3$ & 393,00 & 37.1 & 8.56 & 0.54 & -0.30 \\
\hline $6 \mathrm{R}-2$ & 392.00 & 38.0 & 8.62 & 0.44 & -0.20 \\
\hline $8 R-1$ & 410.00 & 39.9 & 8.84 & 0.52 & 0.10 \\
\hline
\end{tabular}


Table 1 (continued).

\begin{tabular}{|c|c|c|c|c|c|}
\hline $\begin{array}{l}\text { Core. } \\
\text { section }\end{array}$ & $\begin{array}{l}\text { Depth } \\
\text { (mbsf) }\end{array}$ & $\begin{array}{c}\text { Porosity } \\
\text { (\%) }\end{array}$ & $\begin{array}{l}\text { Mean } \\
\text { (phi) }\end{array}$ & Sorting & Skewness \\
\hline IOR-1 & 429.00 & 35.5 & 8.57 & 0.54 & -0.20 \\
\hline $10 R-1$ & 4.30 .00 & 38.3 & 8.52 & 0.41 & $-0,40$ \\
\hline IIR-I & 4.39 .00 & 34.0 & 8.51 & 0.45 & -0.40 \\
\hline IIR-2 & 441.00 & 31.8 & 8.70 & 0.46 & -1.40 \\
\hline $13 R-1$ & 458.00 & 38.6 & 8.86 & 0.50 & 0.16 \\
\hline $13 R-2$ & 460.00 & 38,2 & 8.73 & 0.60 & 0,00 \\
\hline I.3R-3 & 461.00 & 38.5 & 8.60 & 0.55 & -0.20 \\
\hline I4R-1 & 468.00 & 40.0 & 9.14 & 0.50 & 0.10 \\
\hline 14R-CC & 469.00 & 37.8 & 8.71 & 0.33 & 0.00 \\
\hline $15 R-1$ & 477.00 & 39.8 & 8.39 & 0.50 & -0.40 \\
\hline $15 R-2$ & 478.00 & 39.5 & 8.91 & 0.49 & 0.2 .3 \\
\hline \multicolumn{6}{|l|}{$141-863 \mathrm{~A}-$} \\
\hline $1 \mathrm{H}-1$ & 0.07 & 49.8 & 9.03 & 0.54 & 0.19 \\
\hline $\mid \mathrm{H}-1$ & 1.39 & 55.7 & 9.14 & 0.53 & 0.04 \\
\hline IH-2 & 1.91 & 50.5 & 8.99 & 0.49 & 0.30 \\
\hline IH-2 & 2.82 & 50.8 & 9.07 & 0.52 & 0.21 \\
\hline $\mathrm{IH}-3$ & 3.52 & 53.6 & 9.07 & 0.56 & 0.11 \\
\hline $\mathrm{IH}-3$ & 3.98 & 47.8 & 9.07 & 0.54 & 0.14 \\
\hline IH-4 & 4.69 & & 9.03 & 0.53 & 0.21 \\
\hline IH-4 & 5.28 & & 9.03 & 0.51 & 0.24 \\
\hline $1 \mathrm{H}-5$ & 6.41 & 49.5 & 8.90 & 0.62 & 0.06 \\
\hline $1 \mathrm{H}-6$ & 8.03 & 49.7 & 8.96 & 0.53 & 0.20 \\
\hline $\mathrm{IH}-\mathrm{CC}$ & 8.48 & 49.9 & 8.77 & 0.54 & -0.01 \\
\hline $2 \mathrm{H}-\mathrm{I}$ & 9.00 & 49.8 & 8.65 & 0.48 & -0.17 \\
\hline $2 \mathrm{H}-3$ & 11.80 & 47.6 & 8.52 & 0.43 & -0.42 \\
\hline $2 \mathrm{H}-4$ & 13.63 & 43.1 & 8.83 & 0.70 & -0.07 \\
\hline $2 \mathrm{H}-4$ & 14.40 & 45.6 & 8.99 & 0.56 & 0.16 \\
\hline $2 \mathrm{H}-5$ & 15.20 & 52.4 & 8.62 & 0.48 & -0.26 \\
\hline $3 \mathrm{H}-1$ & 18.18 & 48.7 & 8.72 & 0.51 & -0.08 \\
\hline $3 \mathrm{H}-2$ & 20.44 & 51.8 & 8.70 & 0.50 & $-0.30 \$$ \\
\hline $3 \mathrm{H}-2$ & 21.03 & 55.1 & 8.66 & 0.40 & -0.23 \\
\hline $3 \mathrm{H}-3$ & 21.30 & 53.6 & 8.84 & 0,53 & 0.10 \\
\hline $3 \mathrm{H}-3$ & 21.87 & 51.2 & 8.42 & 0.55 & -0.48 \\
\hline $3 \mathrm{H}-4$ & 23.60 & 43.7 & 8.60 & 0.53 & $-0.37 \$$ \\
\hline $3 \mathrm{H}-4$ & 22.81 & 48.5 & 8.36 & 0.56 & -0.47 \\
\hline $3 \mathrm{H}-5$ & 24.96 & 44.8 & 8. & 0.50 & -0.23 \\
\hline $3 \mathrm{H}-6$ & 25.91 & 44.7 & 8.99 & 0.57 & 0.16 \\
\hline $4 \mathrm{H}-1$ & 28.65 & 47.1 & 8.88 & 0.62 & 0.06 \\
\hline $4 \mathrm{H}-2$ & 30.40 & 45.5 & 8.53 & 0.45 & -0.47 \\
\hline $4 \mathrm{H}-3$ & 30.87 & 42.7 & 8.55 & 0.44 & -0.48 \\
\hline $4 \mathrm{H}-\mathrm{CC}$ & 31.35 & 45.0 & 8.55 & 0.42 & -0.45 \\
\hline$S \mathrm{H}-1$ & 38.10 & 45,4 & 8.68 & 0.48 & -0.15 \\
\hline $5 \mathrm{H}-2$ & 39.87 & 43,4 & 8.85 & 0.45 & $-0.19 \S$ \\
\hline $5 \mathrm{H}-2$ & 39.10 & 47.7 & 9.02 & 0.52 & 0.23 \\
\hline SH-CC & 40.37 & 45.4 & 9.02 & 0.51 & 0.25 \\
\hline $6 \mathrm{H}-1$ & 47.10 & 44.8 & 8.92 & 0.52 & 0.22 \\
\hline $6 \mathrm{H}-1$ & 47.84 & 46.5 & 9.11 & 0.52 & 0.14 \\
\hline $7 X-1$ & 57.10 & 46.5 & 9,00 & 0.31 & $-0.07 \$$ \\
\hline $7 \times-2$ & 58.60 & 46.8 & 8.81 & 0.42 & $0.13^{3}$ \\
\hline $7 X-3$ & 60.10 & 47.3 & 8.76 & 0.43 & $-0.11 \$$ \\
\hline $7 \times-4$ & .25 & 50.0 & 8.93 & 0.47 & 0.28 \\
\hline $7 X-5$ & 62.68 & 45.9 & 9.01 & 0.54 & 0.20 \\
\hline $8 X-1$ & 66.18 & 49.3 & 9.04 & 0.60 & 0.05 \\
\hline $8 X-2$ & 68.00 & 48.4 & 8.67 & 0.56 & -0.18 \\
\hline $8 \times-3$ & 69.80 & 42.9 & 8.45 & 0.92 & -0.33 \\
\hline $9 \mathrm{X}-\mathrm{CC}$ & 76.19 & 40.3 & 8.65 & 0.50 & -0.23 \\
\hline $14 X-1$ & 124.10 & 31.5 & 8.78 & 1.03 & $-0.43 \$$ \\
\hline $14 X-2$ & 125.10 & 34.4 & 8.66 & 0.79 & -0.24 \\
\hline $15 \mathrm{X}-\mathrm{CC}$ & 133.50 & 32.0 & 8.60 & 0.70 & $-0.45 \$$ \\
\hline $16 \times-C C$ & 143.10 & 35.0 & 8.62 & 0.57 & -0.27 \\
\hline $17 X-1$ & 152.90 & 31.4 & 8.90 & 1.12 & $-0.59 \$$ \\
\hline $18 \mathrm{X}-\mathrm{CC}$ & 162.50 & 29.1 & 8.75 & 0.70 & -0.14 \\
\hline $19 X-1$ & 172.10 & 33.1 & 8.77 & 0.65 & $-0.49 \$$ \\
\hline $21 X-1$ & 191.40 & 36.6 & 8.89 & 0.47 & 0.25 \\
\hline $21 \mathrm{X}-\mathrm{CC}$ & 191.90 & 41.0 & 8,98 & 0.60 & 0.09 \\
\hline $22 \mathrm{X}-\mathrm{CC}$ & 201.10 & 39.0 & 8.51 & 1.26 & $-0.66 \$$ \\
\hline $23 \mathrm{X}-\mathrm{CC}$ & 211.00 & 38.7 & 7.36 & 2.22 & $-0.86 \$$ \\
\hline $24 X-1$ & 220.70 & 45.5 & 8.55 & 0.71 & -0.27 \\
\hline $24 \mathrm{X}-\mathrm{CC}$ & 221.30 & 33.2 & 7.17 & 2.30 & $-0.82 \leqslant$ \\
\hline $25 \times-1$ & 229,90 & 40.5 & 8.20 & 1.36 & -0.555 \\
\hline $25 x-2$ & 230.40 & 36.4 & 7.16 & 2.32 & $-0.80 \mathrm{~s}$ \\
\hline $25 \mathrm{X}-\mathrm{CC}$ & 231.20 & 40.2 & 8.59 & 1.21 & $-0.63 \xi$ \\
\hline $26 \mathrm{X}-1$ & 239.80 & 44.1 & 9.01 & 0.53 & 0.20 \\
\hline $26 \mathrm{X}-2$ & 240.40 & 39.3 & 8.98 & 0.59 & 0.11 \\
\hline $27 \mathrm{X}-1$ & 249.60 & 45.1 & 8.63 & 0.49 & -0.21 \\
\hline $27 \mathrm{X}-\mathrm{CC}$ & 250.60 & 41.3 & 8.58 & 1.16 & $-0.62 \$$ \\
\hline $28 \mathrm{X}-1$ & 259.00 & 34.2 & 8.19 & 1.43 & $-0.70 \$$ \\
\hline $28 \mathrm{X}-\mathrm{CC}$ & 260.40 & 36.8 & 6.88 & 2.25 & $-0.63 \$$ \\
\hline $29 X-1$ & 268.90 & 37.9 & 8.80 & 0.80 & $-0.16^{\circ}$ \\
\hline $29 X-2$ & 269.60 & 35.6 & 6.96 & 2.24 & $-0.66 \$$ \\
\hline $30 \times-1$ & 279.30 & 39.1 & 8.22 & 1.45 & -0.615 \\
\hline $30 \times-2$ & 280.30 & 39.3 & 6.41 & 2.24 & $-0.24 \$$ \\
\hline $31 \times-1$ & 287.90 & 40.5 & 8.59 & 0.80 & -0.23 \\
\hline $31 \times-C C$ & 288.50 & 38.7 & 8.68 & 0.61 & -0.14 \\
\hline $\begin{array}{c}41-86.3 B \\
4 X-1\end{array}$ & 326.40 & 38.0 & 8.81 & 0.66 & -0.04 \\
\hline
\end{tabular}

\begin{tabular}{|c|c|c|c|c|c|}
\hline $\begin{array}{l}\text { Core, } \\
\text { section }\end{array}$ & $\begin{array}{l}\text { Depth } \\
\text { (mbsf) }\end{array}$ & $\begin{array}{l}\text { Porosity } \\
\text { (C/) }\end{array}$ & $\begin{array}{l}\text { Mean } \\
\text { (phi) }\end{array}$ & Sorting & Skewnes \\
\hline $4 X-2$ & 327.50 & 39.2 & 8.90 & 0.59 & 0.09 \\
\hline $4 X-3$ & 329.90 & 37.1 & 7.08 & 2.30 & $-0.79 \S$ \\
\hline $4 \mathrm{X}-\mathrm{CC}$ & 332.80 & 37.7 & 8.41 & 1.30 & $-0.66 \$$ \\
\hline $5 \mathrm{X}-\mathrm{CC}$ & 335.80 & 32.3 & 8.69 & 0.63 & -0.13 \\
\hline $6 \mathrm{X}-\mathrm{CC}$ & 345.50 & 36.1 & 8.88 & 0.78 & -0.16 \\
\hline $7 \mathrm{~N}-1$ & 355.90 & 40.0 & 8.93 & 0.49 & 0.25 \\
\hline $7 \mathrm{~N}-1$ & 356.10 & 38.3 & 8.49 & 0.51 & -0.52 \\
\hline $7 \mathrm{~N}-2$ & 357.20 & 37.7 & 8.57 & 0.57 & -0.32 \\
\hline $8 \mathrm{~N}-1$ & 357.60 & 31.4 & 6.99 & 2.48 & $-0.81 \$$ \\
\hline $8 \mathrm{~N}-2$ & 358.40 & 40.1 & 8.74 & 0.56 & $-0.29 \leqslant$ \\
\hline $9 \mathrm{X}-\mathrm{CC}$ & 3.70 & 36.0 & 8.77 & 0.67 & -0.08 \\
\hline $10 R-1$ & 372.00 & 39.6 & 8.90 & 0.51 & 0.19 \\
\hline $10 \mathrm{R}-1$ & 371.10 & 36.3 & 8.53 & 0.81 & -0.33 \\
\hline $10 R-1$ & 371.60 & 9.4 & 4.80 & 2.66 & 0.60 \\
\hline $10 \mathrm{R}-1$ & & & 8.72 & 0.65 & -0.17 \\
\hline $\mid 1 R-1$ & 377.10 & 39.9 & 8.54 & 0.51 & -0.54 \\
\hline IIR-I & & & 8.36 & 0. & -0.59 \\
\hline IIR-I & 376.60 & 40.8 & 8.60 & 0.55 & -0.37 \\
\hline $12 \mathrm{R}-1$ & 386.30 & 38.1 & 8.90 & 0.51 & 0.19 \\
\hline $12 \mathrm{R}-1$ & 386.70 & 7.7 & 5.99 & 2.82 & $-0.33 \xi$ \\
\hline 13R-1 & 395.90 & 37.4 & 8.91 & 0.53 & 0.15 \\
\hline $13 R-1$ & 396.40 & 37.5 & 8.68 & 0.71 & $-0.51 \S$ \\
\hline $14 \mathrm{R}-1$ & 406.40 & 38.6 & 8.52 & 0.74 & -0.34 \\
\hline $14 R-2$ & 407.50 & 34.8 & 8.75 & 0.51 & -0.06 \\
\hline $14 R-3$ & 408.40 & 33,4 & 8.77 & 1.13 & $-0.56 \$$ \\
\hline $15 \mathrm{R}-1$ & 416.50 & 34.5 & 7.00 & 2.35 & $-0.78 \$$ \\
\hline $15 R-1$ & 415,60 & 32.4 & 8.73 & 0.67 & $-0.09^{\circ}$ \\
\hline $15 \mathrm{R}-2$ & 417.80 & 32.4 & 8.95 & 0.69 & -0.07 \\
\hline $15 \mathrm{R}-\mathrm{CC}$ & 418.20 & 34.4 & 8.70 & 0.80 & -0.17 \\
\hline $16 \mathrm{R}-1$ & 425.50 & 36.7 & 9.02 & 0.70 & -0.11 \\
\hline $16 \mathrm{R}-2$ & 426.80 & 33.9 & 6.61 & 2.28 & $-0.54 \$$ \\
\hline $16 R-3$ & 428.70 & 31.0 & 8.27 & 1.21 & $-0.71 \S$ \\
\hline $16 R-4$ & 430.50 & 10.5 & 5.13 & 2.58 & $0.59 \$$ \\
\hline $16 R-5$ & 431.10 & 29.4 & 6.70 & 2.56 & $-0.80 \leqslant$ \\
\hline $17 \mathrm{R}-1$ & 434.50 & 29.3 & 5.27 & 2.50 & $0.62 \$$ \\
\hline $17 \mathrm{R}-2$ & 436.10 & 30.4 & 7.95 & 1.37 & $-0.60 \$$ \\
\hline $17 R-5$ & 441.40 & 27.0 & 6.81 & 2.33 & $-0.72 \S$ \\
\hline $17 \mathrm{R}-7$ & 443.30 & 27.6 & 6.66 & 2.65 & -0.838 \\
\hline I8R-1 & 444.20 & 29.0 & 8.70 & 0.62 & -0.15 \\
\hline 18R-2 & 445,60 & 27.9 & 8.37 & 0.59 & $0.77 \S$ \\
\hline $18 \mathrm{R}-4$ & 448.70 & 30.2 & 8.20 & 0.54 & $-0.66 \$$ \\
\hline 18R-2 & 446.50 & 34.8 & 8.64 & 0.51 & -0.21 \\
\hline $19 R-1$ & 455.10 & 34.0 & 8.79 & 0.56 & -0.01 \\
\hline $19 \mathrm{R}-3$ & 457.10 & 30.5 & 6.07 & 2.52 & $-0.21 \S$ \\
\hline 19R-5 & 459.40 & 30.2 & 8.52 & 0.59 & $-0.49 \$$ \\
\hline $20 \mathrm{R}-1$ & 464.40 & 29.3 & 8.65 & 0.54 & $-0.29 \$$ \\
\hline $20 \mathrm{R}-2$ & 465.40 & 28.6 & 8.76 & 1.14 & -0.628 \\
\hline $20 \mathrm{R}-3$ & 466.50 & 24.0 & 8.23 & 0.81 & $-0.84 \$$ \\
\hline $2 \mid R-1$ & 473.60 & 32.3 & 6.47 & 2.24 & $-0.63 \S$ \\
\hline $2 \mid \mathrm{R}-2$ & 474.90 & 30.4 & 6.37 & 2.38 & $-0.47 \$$ \\
\hline $2 \mathrm{IR}-\overline{C C}$ & 475.40 & 34.1 & 7.52 & 1.58 & $-0.84 \$$ \\
\hline $22 \mathrm{R}-1$ & 483.80 & 27.8 & 7.01 & 2.14 & $-0.87 \$$ \\
\hline $22 \mathrm{R}-2$ & 484.80 & 29.8 & 8.36 & 0.55 & -0.46 \\
\hline $22 \mathrm{R}-3$ & 485.80 & 29.1 & 8.32 & 0.59 & -0.49 \\
\hline $23 R-1$ & 493.00 & 25.3 & 8.50 & 0.45 & -0.43 \\
\hline $23 \mathrm{R}-4$ & 497.90 & 24.5 & 8.23 & 0.64 & -0.43 \\
\hline $24 \mathrm{R}-1$ & 503.30 & 29.0 & 8.59 & 0.57 & -0.42 \\
\hline $24 \mathrm{R}-2$ & 503.70 & 29.8 & 6.75 & 2.23 & $-0.68 \S$ \\
\hline $24 \mathrm{R}-3$ & 506.20 & 29.8 & 8.57 & 0.67 & $-0.44 \$$ \\
\hline $24 \mathrm{R}-4$ & 506.80 & 27.0 & 8.16 & 1.05 & $-0.75 \$$ \\
\hline $25 \mathrm{R}-2$ & 514.20 & 27.7 & 6.41 & 2.25 & -0.588 \\
\hline $25 \mathrm{R}-1$ & 512.20 & 23.3 & 8.39 & 0.65 & $-0.76 \$$ \\
\hline $25 R-3$ & 515.00 & 29.5 & 6.87 & 2.23 & $-0.68 \$$ \\
\hline $26 R-1$ & 521.40 & 10.8 & 7.09 & 2.34 & $-0.77 \$$ \\
\hline $26 R-2$ & 522.80 & 21.0 & 8.41 & 0.47 & -0.698 \\
\hline $27 R-4$ & 535.90 & 30.1 & 6.77 & 2.31 & $-0.69 \$$ \\
\hline $27 \mathrm{R}-1$ & 530.90 & 29.8 & 8.73 & 1.13 & $-0.60 \$$ \\
\hline $27 \mathrm{R}-3$ & 534.40 & 28.7 & 8.18 & 1.24 & $-0.74 \$$ \\
\hline $28 R-2$ & 541.30 & 32.1 & 6.98 & 2.36 & -0.758 \\
\hline $28 \mathrm{R}-2$ & 541.50 & 27.6 & 8.00 & 0.72 & -0.33 \\
\hline $28 R-1$ & 540.70 & 28.7 & 8.35 & 0.67 & -0.56 \\
\hline 29R-CC & 549.70 & 27.6 & 8.45 & 0.64 & -0.39 \\
\hline $3 \mid R-1$ & 569,30 & 24.4 & 8.86 & 0.73 & -0.08 \\
\hline 3 IR-5 & 574.80 & 21.5 & 8.38 & 0.62 & -0.49 \\
\hline $3 \mid \mathrm{R}-1$ & 569.50 & 24.3 & 6.95 & 2.26 & $-0.76 \S$ \\
\hline $32 \mathrm{R}-1$ & 579.80 & 24.8 & 8.61 & 0.51 & -0.27 \\
\hline $32 \mathrm{R}-4$ & 584.40 & 31.7 & 8.35 & 0.57 & -0.48 \\
\hline $32 \mathrm{R}-6$ & 586.70 & 27.9 & 8.57 & 0.52 & -0.34 \\
\hline $32 \mathrm{R}-2$ & 581.60 & 27.5 & 8.59 & 0.58 & -0.378 \\
\hline $32 \mathrm{R}-\mathrm{CC}$ & 587.90 & 31.2 & 8.33 & 0.62 & -0.55 \\
\hline $33 R-2$ & 591.00 & 27.3 & 8.32 & 1.14 & $-0.65 \S$ \\
\hline $33 R-1$ & 589.20 & 25.9 & 7.04 & 2.39 & $-0.80 \leqslant$ \\
\hline $33 R-3$ & 591.30 & 23.7 & 8.26 & 1.23 & -0.718 \\
\hline $33 R-4$ & 592.20 & 25.6 & 8.59 & 0.58 & $-0.26^{\circ}$ \\
\hline $34 \mathrm{R}-1$ & 598.30 & 26.9 & 8.37 & 1.28 & $-0.60 \$$ \\
\hline $34 \mathrm{R}-6$ & 06.30 & 25.7 & 8.27 & 1.40 & $-0.65 \$$ \\
\hline $34 \mathrm{R}-2$ & 600.20 & 23.8 & 8.30 & 0.63 & -0.47 \\
\hline
\end{tabular}


Table 1 (continued).

\begin{tabular}{|c|c|c|c|c|c|}
\hline $\begin{array}{l}\text { Core. } \\
\text { section }\end{array}$ & $\begin{array}{l}\text { Depth } \\
\text { (mbsf) }\end{array}$ & $\begin{array}{c}\text { Porosity } \\
\text { (\%) }\end{array}$ & $\begin{array}{l}\text { Mean } \\
\text { (phi) }\end{array}$ & Sorting & Skewness \\
\hline $34 \mathrm{R}-4$ & 603.20 & 22.0 & 7.98 & 0.81 & -0.32 \\
\hline $35 R-3$ & 611.10 & 24.4 & 8.58 & 0.61 & -0.26 \\
\hline $35 R-1$ & 609.10 & 24.1 & 8.16 & 1.31 & $-0.73 \S$ \\
\hline $35 \mathrm{R}-5$ & 613.90 & 2.3 .9 & 8.28 & 0.74 & -0.48 \\
\hline $35 \mathrm{R}-\mathrm{CC}$ & 616.10 & 26.1 & 8.42 & 0.86 & -0.37 \\
\hline $36 \mathrm{R}-3$ & 620.40 & 19.4 & 8.37 & 0.78 & -0.37 \\
\hline $36 \mathrm{R}-1$ & 617.90 & 27.9 & 8.15 & 0.85 & $-0.41 \S$ \\
\hline $36 R-2$ & 619.20 & 21.8 & 8.57 & 0.73 & -0.24 \\
\hline $37 \mathrm{R}-1$ & 628.20 & 22.4 & 8.01 & 1.28 & -0.69 \\
\hline $37 \mathrm{R}-4$ & 6.32 .30 & 24.6 & 6.80 & 2.49 & $-0.69 \S$ \\
\hline $37 R-5$ & 6.34 .40 & 23.2 & 7.07 & 2.20 & $-0.80 \$$ \\
\hline $38 \mathrm{R}-1$ & 637.10 & 24.0 & 8.81 & 0.75 & -0.12 \\
\hline $38 \mathrm{R}-2$ & 6.38 .70 & 22.3 & 8.13 & 0.81 & $-0.36 \$$ \\
\hline $38 \mathrm{R}-2$ & 639.20 & 22.0 & 8.43 & 0.51 & -0.40 \\
\hline $39 \mathrm{R}-2$ & 648.40 & 28.5 & 6.86 & 2.39 & $-0.67 \S$ \\
\hline $39 \mathrm{R}-3$ & 650.00 & 21.3 & 8.27 & 1.28 & $-0.68 \S$ \\
\hline $40 \mathrm{R}-1$ & 656.20 & 24.3 & 8.01 & 1.27 & $-0.50 \$$ \\
\hline $40 \mathrm{R}-3$ & 659.00 & 26.1 & 8.36 & 0.79 & -0.43 \\
\hline $4 \mid R-1$ & 665.90 & 19.4 & 8.26 & 0.68 & -0.54 \\
\hline $4 I R-2$ & 667.20 & 23.5 & 5.70 & 2.38 & $0.10 \leqslant$ \\
\hline $4 \mid R-4$ & 670.30 & 22.6 & 8.42 & 0.55 & -0.53 \\
\hline $42 \mathrm{R}-2$ & 677.00 & 21.4 & 7.64 & 1.61 & $-0.69 \S$ \\
\hline $42 \mathrm{R}-1$ & 676.30 & 19.6 & 8.68 & 0.62 & $-0.44 \S$ \\
\hline $42 \mathrm{R}-3$ & 678.40 & 19.6 & 8.27 & 0.67 & -0.55 \\
\hline $43 \mathrm{R}-1$ & 685.50 & 20.0 & 8.20 & 0.61 & -0.33 \\
\hline $43 R-2$ & 686.90 & 24.3 & 8.52 & 0.49 & -0.43 \\
\hline $44 R-2$ & 696.90 & 24.6 & 8.32 & 0.66 & -0.58 \\
\hline $44 R-3$ & 698.70 & 26.0 & 7.14 & 2.21 & $-0.75 \S$ \\
\hline $44 \mathrm{R}-4$ & 699.50 & 24.0 & 8.13 & 0.82 & $-0.37 \S$ \\
\hline $45 \mathrm{R}-1$ & 704.90 & 28.1 & 8.14 & 0.62 & $-0.22 \S$ \\
\hline $46 \mathrm{R}-1$ & 708.00 & 28.2 & 8.54 & 0.51 & -0.43 \\
\hline $46 \mathrm{R}-2$ & 708.90 & 23.0 & 7.95 & 1.48 & $-0.82 \S$ \\
\hline $46 \mathrm{R}-2$ & 709.00 & 22.7 & 8.86 & 0.56 & 0.08 \\
\hline $47 R-1$ & 714.90 & 25.2 & 5.45 & 2.34 & $0.33 \S$ \\
\hline $47 \mathrm{R}-2$ & 715.40 & 22.6 & 5.21 & 2.42 & $0.77 \S$ \\
\hline $47 R-2$ & 716.00 & 26.0 & 8.39 & 0.52 & $-0.14 \$$ \\
\hline $47 R-3$ & 717.50 & 26.5 & 7.66 & 0.61 & -0.25 \\
\hline $48 \mathrm{R}-2$ & 724.20 & 24.4 & 6.90 & 2.15 & $-0.77 \S$ \\
\hline $48 \mathrm{R}-2$ & 724.50 & 24.7 & 8.20 & 0.74 & $-0.39 \$$ \\
\hline $49 \mathrm{R}-1$ & 733.70 & 25.5 & 6.80 & 2.41 & $-0.75 \$$ \\
\hline $49 \mathrm{R}-1$ & 734.60 & 20.1 & 8.45 & 0.82 & $-0.51 \S$ \\
\hline $49 R-4$ & 737.80 & 21.8 & 8.28 & 0.59 & $-0.60 \$$ \\
\hline
\end{tabular}

Note: The textural data were produced by a laser particle counter and a rapid sediment analyzer 442 nonsandy samples were measured by the laser particle counter only. 129 sandy samples were measured by both instruments and the grain-size distribution data merged. These samples are indicated by the superscript $(\$)$ symbol. The data from the 129 sandy samples were not used in Figures 3-7.

by the Department of Geology at Franklin and Marshall College. The RSA consists of a 1.5 -m-long by 20 -cm-diameter acrylic tube with a weighing pan suspended at the bottom of the tube. The pan is attached by monofilament line to a digital balance above the tube. The balance is capable of measuring weights to the nearest $0.0001 \mathrm{~g}$. The sample is introduced into the top of the tube by means of an injection plate coated with photo-flow solution. The surface tension of the photoflow holds the approximately 0.25 -g sample of sand-size material even when the plate is inverted. When the plate is lowered into the top of the water column, the photo-flow dissolves and releases the grains. The contact of the injection plate with the water also triggers the balance and computer to begin the analysis. The rate at which mass accumulates on the weighing pan is converted into grain-size distributions by means of the Gibbs equation (cf., Halka, Conkwright, Kerhin, and Wells, 1980). This data is then plotted on cumulative frequency curves to determine the $95 \%, 84 \%, 50 \%, 16 \%$, and $5 \%$ values from which the mean, sorting and skewness can be calculated (Carver, 1971; Folk, 1974; Leeder, 1982; McManus, 1988).

The samples that most likely contained sand were identified using the shipboard logs from Leg 141. Smear slides of those samples were examined under a petrographic microscope to estimate the abundance of particles coarser than $64 \mu \mathrm{m}$. Samples that had significant quantities of sand-size material were prepared for the RSA by wet sieving.
Samples of 1 to $5 \mathrm{~g}$ were placed in mortars and soaked for several hours in distilled water containing a small amount of sodium hexametaphosphate (Carver, 1971). The samples were gently disaggregated using finger pressure or, when necessary, a pestle. The samples were then wet sieved using distilled water to remove material finer than 4 phi. The product was checked with a binocular microscope to ensure that the clay and silt fractions had been removed. The sandsize material was trapped on filter paper, dried, and stored until analyzed. The weight of the sand sample was compared to the starting weight of the bulk sample in order to determine the weight percent sand in the sandy samples.

The grain-size distribution data for the 129 sandy samples measured by the RSA are incorporated into Table 1 and indicated by a superscript symbol. These samples generally represent 1 to $10 \mathrm{wt} \%$ of the starting samples, although a number of samples exceed $10 \%$ and a few are composed almost entirely of sand (more than 90\%). Holes $859 \mathrm{~A}$ and $859 \mathrm{~B}$ did not contain sufficient sandy material to warrant RSA analysis. Replicate runs of individual samples produced comparable results.

\section{RESULTS}

The grain-size distribution data for the 442 non-sandy samples (laser particle counter) and the 129 sandy samples (combined laser particle counter and RSA, indicated by the superscript symbol) appear in Table 1 . The data for the 442 non-sandy samples were generated by only one analytical technique and were therefore used for further analysis. These data are plotted in a series of diagrams that show (1) the change of porosity, mean size, and sorting as functions of burial, (2) the degree of sorting as a function of mean grain size, and (3) the changes in sorting and mean size as functions of the skewness of the grain-size populations (Figs. 3 through 7). Figure 7 is a cumulative plot of (1) sorting versus mean grain size and (2) sorting and mean grain size versus skewness for all of the sites. The details of the results are discussed below for each site.

\section{Site 859}

Mean grain size ranges between 8.7 and 9.3 phi with an average of around 9 phi in the nearly $500 \mathrm{~m}$ of sediment recovered from Site 859 (Fig. 3A). The largest mean grain size (maxima) of 8.7 phi occurs at about $400 \mathrm{mbsf}$. Other than this, grain size remains uniform downhole, even where the samples become more indurated, possibly reflecting an increased degree of cementation (Behrmann, Lewis, Musgrave, et al., 1992). Thus, the grain-size data resulting from the sample preparation and analysis techniques used in this study do not seem to be affected by cements. Also apparent from Figure $3 \mathrm{~A}$ is a trend of progressive decrease of porosity with depth below seafloor. Near the surface, wet porosity values are in the $50 \%$ to $60 \%$ range (Behrmann, Lewis, Musgrave, et al., 1992). At the base of the site (about $470 \mathrm{mbsf}$ ) porosities are reduced to a minimum of $10 \%$. There are several localized reversals of this trend: at about $50-80,200,380-415$, and more than $460 \mathrm{mbsf}$, where porosities are greater than expected. All of these, with the possible exception of the interval around $200 \mathrm{~m}$, may be related to the slightly coarser mean grain size at those intervals.

The relationship between sorting and porosity with depth below seafloor are illustrated in Figure 3B (the porosity versus depth plot is repeated from Fig. $3 \mathrm{~A}$ ). Sorting values range between 0.15 (very well sorted) and 0.6 (moderately well sorted) with most of the samples clustered around 0.5 (well sorted to moderately well sorted). One zone of very well sorted samples occurs at about $400 \mathrm{mbsf}$, at the same depth where porosity exhibits locally high values. There does not appear to be a similar correlation at $200 \mathrm{mbsf}$, however, because sorting values at that depth are about 0.5 (about average for Site 859).

There are some interesting relationships between sorting and mean grain size at Site 859, which are illustrated in Figure 3C. The sizes range between about 8.7 and 9.3 phi. Although sorting varies between about 0.15 (very well sorted) and 0.6 (moderately well sorted), the 
A
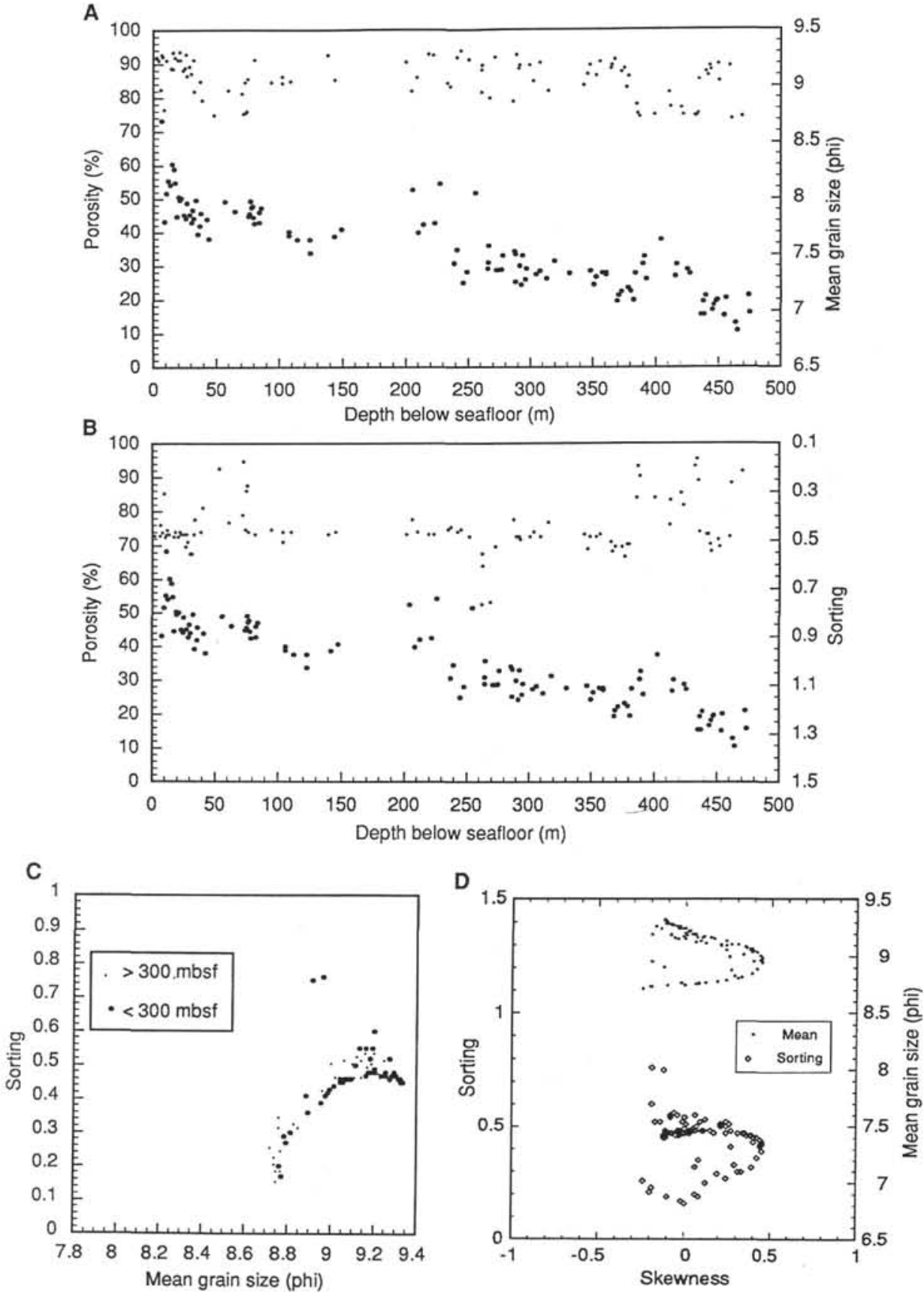

Figure 3. Textural and porosity data for non-sandy samples analyzed by the laser particle counter, Site 859. A. Porosity (large dots) and mean grain size (small dots) vs. depth. B. Porosity (large dots) and sorting (small dots) vs. depth. C. Sorting vs. mean grain size. D. Sorting and mean grain size vs. skewness.

coarsest material at Site 859 is the best sorted material. Overall, there is a trend toward poorer sorting as grain size decreases. However, for grain sizes finer than 9.2 phi, there is a reversal toward slightly better sorting with decreased grain size. This reversal may be the result of instrument limitations. The laser particle counter has a $1 \mu$ (or 10 phi) lower limit of resolution and all material less than $1 \mu$ in size is classed together for purposes of calculating mean size and sorting. As more of the sample falls into the class finer than 10 phi, the grain-size distribution may become apparently better sorted as a result.

The relationship of mean grain size versus skewness appears in Figure $3 \mathrm{D}$ as a $U$-shaped plot lying on its side. Skewness reaches its most positive values at mean sizes of about 8.9 phi and decreases as mean grain size fines and coarsens away from that value (thereby defining the $U$-shaped plot). The decrease in skewness in the upper limb of the $U$-shaped plot (mean grain-size range of 8.9 to 9.3 phi) may be due to instrument limitation. As the instrument limit of $10 \mathrm{phi}$ is approached, all material finer than 10 phi is lumped into one size category, thereby limiting the fine grained tails of the grain-size distributions and producing negative skewness values. The decrease in skewness in the lower limb of the $U$-shaped plot (mean grain-size range of 8.9 to $8.6 \mathrm{phi}$ ) may be due to the presence of a wide range of coarser grains in material of progressively coarser mean grain size. The relationship of skewness to sorting also appears in Figure 3D. Skewness achieves it most positive value for a sorting of about 0.4 . With a progressive decrease in skewness the sorting values occur in an increasingly wider range from about 0.15 to 0.8 . 


\section{Site 860}

The variation of porosity and mean grain size versus depth below seafloor for $610 \mathrm{~m}$ of section at Site 860 are illustrated in Figure 4A. The mean grain size ranges between 8.2 and 9.1 phi and averages about 8.8 phi. It is worth noting that mean grain size appears to exhibit cyclic changes (oscillations) with depth below seafloor. The coarsest grain sizes (maxima) occur at depths of 120-160, 350-410, and 520-540 mbsf. The finest grain sizes (minima) occur at depths of $0-40,110-150,280-320,410-450$, and 540-580 mbsf. The overlap in grain-size maxima and minima between 120 and $150 \mathrm{mbsf}$ is the result of the wide range of mean grain sizes in this interval. Wet porosity decreases progressively with depth below seafloor (Fig. 4A). Porosity near the surface is between $50 \%$ and $60 \%$ and at the base is reduced to about $35 \%$. Local increases in porosity occur at about 110 , 290,440 , and $580 \mathrm{mbsf}$, corresponding to most of the grain-size minima discussed above, which suggests that the finer the grain size, the higher the porosity of these samples. Possible causes for the cyclicity in Figure 4A are discussed below.

The relationship of porosity and sorting versus depth below seafloor for Site 860 are illustrated in Figure 4B. Sorting ranges between 0.2 (very well sorted) and 0.8 (moderately sorted) and appears to become less well sorted with depth. The overall trend of decreased sorting is not a smooth curve, but rather it has an oscillating pattern. Relatively poorly sorted material occurs at depths of about 20-40, $140-170,260,390-420$, and 530 mbsf. These correspond with grainsize maxima and indicate that the coarser material is less well sorted at Site 860 . There does not seem to be a corresponding relationship between relatively well sorted material and grain-size minima, however. Nor is there an obvious relationship between local porosity maxima and either sorting maxima or minima.

An interesting relationship of sorting versus mean grain size for Site 860 is illustrated in Figure 4C. The data plot as a rhomboid pattern in which the best-sorted material (sorting value of 0.2 ) is situated at a grain size of 8.7 phi. The margin of the rhomboid, which extends up to the right, indicates that as grain size fines, sorting decreases. This margin of the rhomboid is similar to the data that appears in Figure 3C (the sorting versus grain-size plot for Site 859). The similarity in the two suggests that this recurring pattern is controlled by similar sedimentary processes. The rhomboid pattern also has a lower limb rising to the left, which shows that as grain size coarsens, sorting diminishes. The presence of the material coarser than 8.7 phi suggests that there may be an additional sedimentary process supplying material at Site 860 compared with the processes operating at Site 859 .

The relationship of sorting and mean grain size versus skewness illustrated in Figure 4D is largely similar to that observed in Figure $3 \mathrm{D}$. One difference is that the upper limb of the $U$-shaped skewness versus mean grain-size plot is much less pronounced, presumably due to the relative lack of very fine grained samples. As in Figure 3D, the lower limb of the $U$-shaped plot exhibits progressively more negative skewness as mean grain size increases.

\section{Site 861}

The variation of porosity and mean grain size with depth below seafloor at Site 861 is illustrated in Figure 5A. Mean grain size ranges from about 8.2 to 9.1 phi and averages about 8.7 phi throughout 500 $\mathrm{m}$ of sediment at Site 861. There appears to be some oscillation of mean grain size with the coarsest sizes (maxima) at depths of about $50,120-130$, and $240-280 \mathrm{mbsf}$. The finest mean grain-size values (minima) occur at depths of about 70-80, 150-190,300-350, and 470 mbsf. Also shown on Figure $5 \mathrm{~A}$ is the change in porosity with depth below seafloor. Near the surface, the porosity is between $50 \%$ and $60 \%$. The porosity diminishes to $30 \%$ to $40 \%$ at the bottom of Site 861 . The overall decrease in porosity appears to occur in steps with notable drops at about 200 and 350 mbsf. Between these notable drops the porosity values remain uniform to slightly decreasing with increasing depth below seafloor. These two notable drops correspond to transitions between grain-size minima and grain-size maxima in the upper curve of Figure 5A. Perhaps as grain size increases, porosity decreases in these two cases.

The change in sorting and porosity with depth below seafloor is illustrated in Figure 5B. Sorting ranges from 0.25 (very well sorted) to 0.75 (moderately sorted) and averages about 0.55 (moderately well sorted). The average value for sorting does not change much with depth, but there are oscillating excursions from the average. Low values (indicating higher degrees of sorting) occur at about 0-30, 230, 350 , and $470 \mathrm{mbsf}$. The high degree of sorting at $350 \mathrm{mbsf}$ corresponds with the notable increase in grain size and reduction of porosity discussed above.

The relationship of sorting to mean grain size for Site 861 is illustrated in Figure 5C. The plot is similar in pattern to the sorting versus mean grain-size plot for Site 860 (Fig. 4C), which suggests that similar sedimentary processes are responsible for the samples at both sites. Some of the samples with a grain size of about 8.7 phi are very well sorted. Sorting diminishes as grain size both fines and coarsens from that value to produce the margins of the rhomboid pattern seen in Figure $5 \mathrm{C}$. On the limb that rises to the right as grain size fines, there is an indication that the sorting values level off at about 9 to 9.15 phi. This may be the result of the instrument-dependent boundary effect discussed for the sorting versus mean grain-size plot for Site 859 .

The relationships of sorting and mean grain size to skewness for Site 861 illustrated in Figure 5D are similar to those in Figure 4D. One difference is that the upper limb of the $U$-shaped plot for skewness versus mean grain size is nearly non-existent.

\section{Site 863}

The relationships of mean grain size and porosity to depth below seafloor for $720 \mathrm{~m}$ of section recovered from Site 863 are illustrated in Figure 6A. Mean grain sizes range from 7.6 to 9.2 phi and average about 8.7 phi in the upper $460 \mathrm{~m}$ of the site. Below $460 \mathrm{mbsf}$, the average grain size is about 8.4 phi. The porosity values illustrated in Figure $6 \mathrm{~A}$ are about $50 \%$ near the surface and diminish to about $25 \%$ at the base. The porosity exhibits a local minima at $160-180 \mathrm{mbsf}$. There is also a marked decline in porosity between 460 and $480 \mathrm{mbsf}$, coincident with the increase in average mean grain size at that level.

The relationship of sorting to depth below seafloor for Site 863 is illustrated in Figure 6B. Sorting ranges from 0.4 (well sorted) to 0.85 (moderately sorted) with an average sorting of about 0.65 (moderately well sorted). With the exception of the upper $100 \mathrm{~m}$, which is on average slightly better sorted, the degree of sorting appears to be uniform throughout Site 863.

The relationship of sorting and mean grain size is illustrated in Figure $6 \mathrm{C}$. The data occur in a rhomboid pattern similar to those seen in Figures $4 \mathrm{C}$ and $5 \mathrm{C}$. The best sorted material is found at a mean grain size of 8.7 phi. However, because of the wider range of grain sizes that have well sorted material, the rhomboid does not have as pronounced a "tail" as Figures $4 \mathrm{C}$ and $5 \mathrm{C}$. Nonetheless, sorting worsens as grains sizes fine and coarsen away from 8.7 phi, again suggesting that similar sedimentary processes are acting at Site 863 as at the other sites. The data from either side of $300 \mathrm{mbsf}$ are uniformly distributed in the rhomboid, suggesting that grain size and sorting are not affected by cement formation.

The relationships between sorting, mean grain size and skewness illustrated in Figure 6D are very similar to those for Figures $4 \mathrm{D}$ and $5 \mathrm{D}$. One difference is that the upper limb of the $U$-shaped plot for skewness versus mean grain size has no expression in Figure 6D. This may be due to the absence of a very fine-grained component in the Site 863 sample set.

\section{Composite Plots for Sites 859, 860, 861, and 863}

The relationship between mean grain size and sorting for all four sites (Sites 859, 860, 861, and 863) is illustrated in Figure 7A. The 

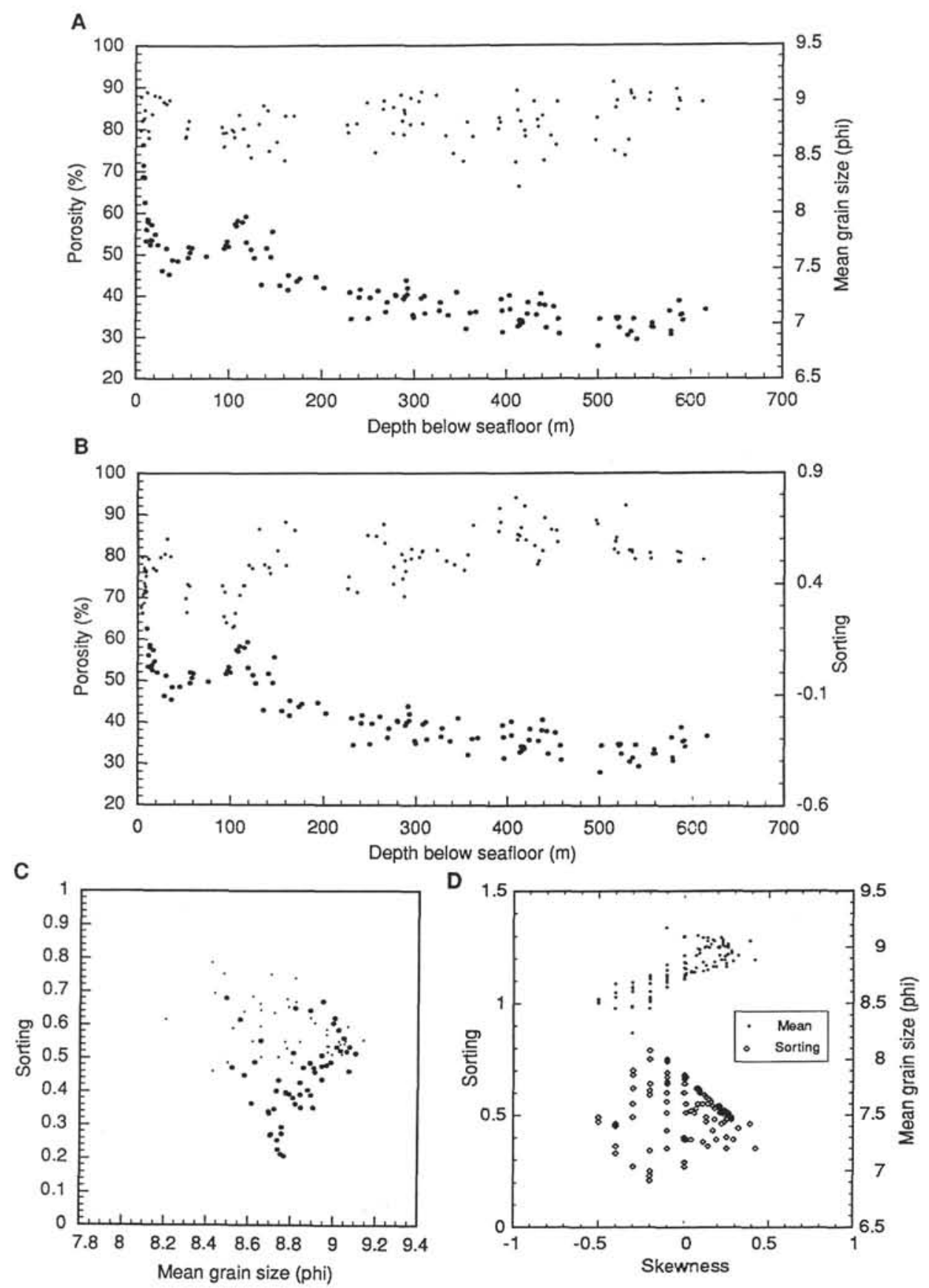

Figure 4. Textural and porosity data for non-sandy samples analyzed by the laser particle counter, Site 860 . A. Porosity and mean grain size vs. depth. B. Porosity and sorting vs. depth. C. Sorting vs. mean grain size. D. Sorting and mean grain size vs, skewness. See Figure 3 for explanation of symbols.

rhomboid pattern is evident with the best-sorted material occurring at about 8.7 phi. Sorting worsens as grain size fines and coarsens away from that value. There is a fairly uniform distribution of values for depths less than $300 \mathrm{mbsf}$ (solid squares) and depths greater than 300 mbsf (diamonds); thus, there seems to be little effect of possible depth-dependent cements on either mean grain size or sorting.

The relationships of sorting and mean grain size to skewness for all four sites is illustrated in Figure 7B. Skewness versus mean grain size for the entire data set exhibits a $U$-shaped pattern where the upper limb (for mean grain sizes from 8.9 to 9.3 phi) may be due to an instrument effect acting on fine-grained samples. The lower limb of the $U$-shaped pattern (for mean grain sizes from 8.9 to 8.3 phi) shows a consistent relationship of decreasing skewness with increasing mean grain size. Sorting versus skewness for the entire data set exhibits the most positive skewness values for sorting values of about 0.4 . As skewness values diminish, the sorting values occur in an increasingly wide range (from 0.9 to 0.2 ).

\section{DISCUSSION}

Grain-size distribution data can provide insights into environments of deposition and sediment diagenesis. Furthermore, physical and chemical properties such as porosity, seismic velocity, and interstitial pore-water chemistry are influenced by depositional and diage- 
A
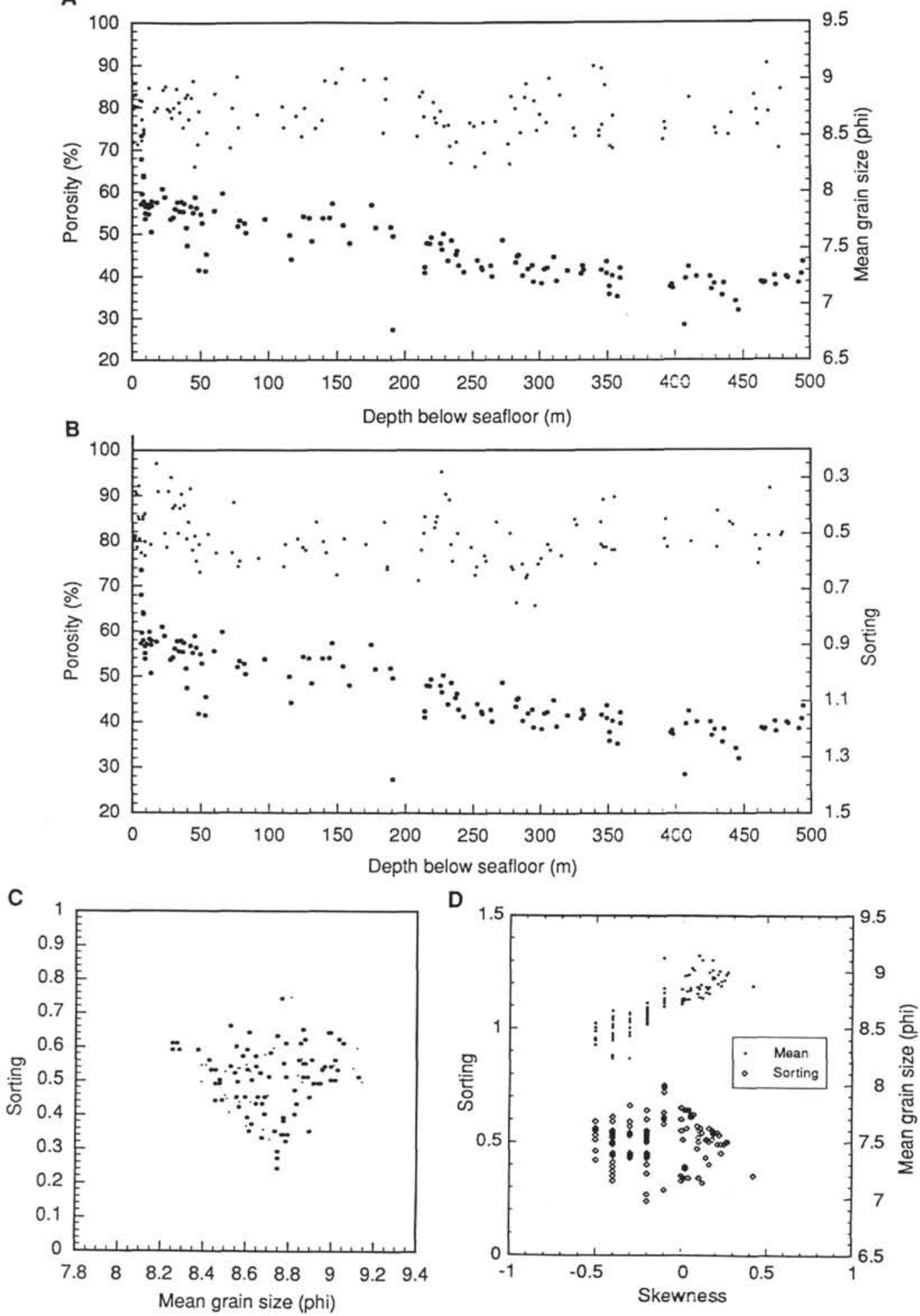

Figure 5. Textural and porosity data for non-sandy samples analyzed by the laser particle counter, Site 861 . A. Porosity and mean grain size vs. depth. B. Porosity and sorting vs. depth. C. Sorting vs. mean grain size. D. Sorting and mean grain size vs. skewness. See Figure 3 for explanation of symbols.

netic conditions related to grain size (Hamilton, 1974; Hein, 1989). Because of the difficulty in visually distinguishing between fine grain sizes, the grain-size data presented here supplements information in the shipboard descriptions of Leg 141 sediments and sedimentary rocks. These additional grain-size data may contribute to our understanding of depositional environments, diagenetic history, and the physical and chemical properties measured aboard the ship.

\section{Depositional Facies Model}

Figure 8 presents a facies model that could account for the analyzed sediments (Behrmann, Lewis, Musgrave, et al., 1992). In this model sediments are supplied to the slope by sediment slumps, debris flows, turbidity flows, suspension fallout from meltwater plumes, hemipelagic sedimentation of organics, and thermohaline (contour) 
A

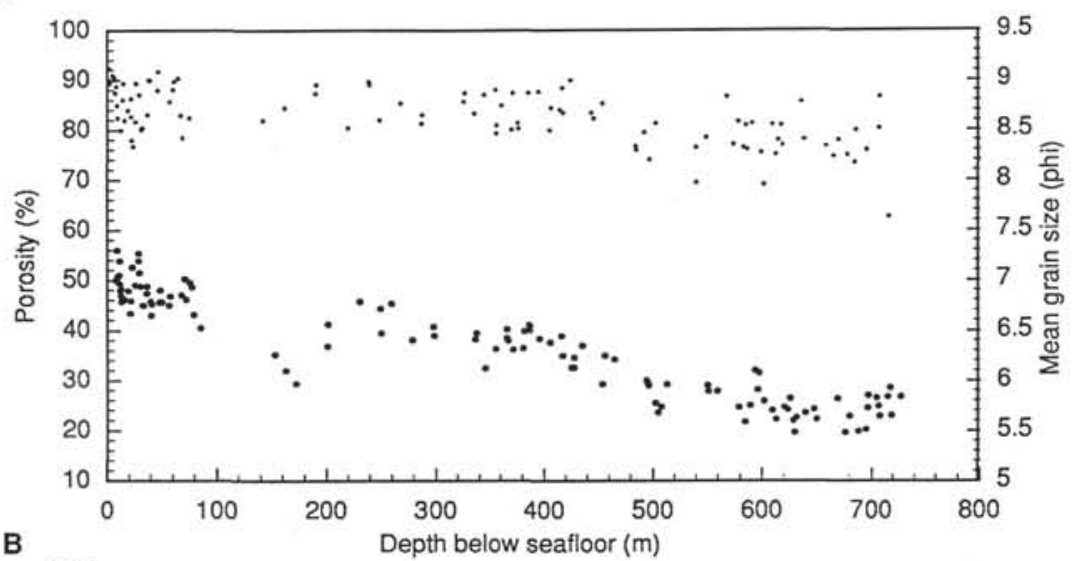

B
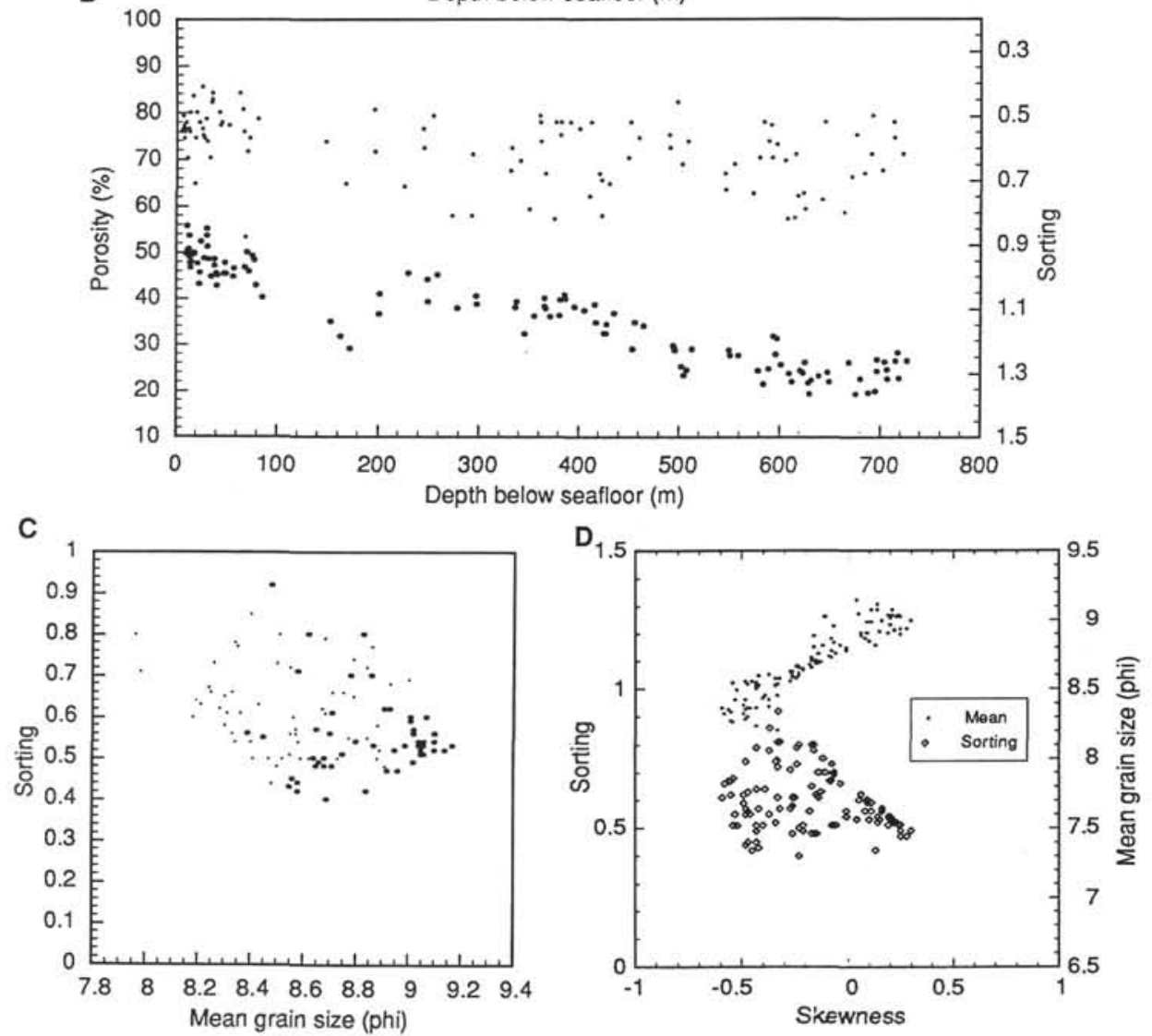

Figure 6. Textural and porosity data for non-sandy samples analyzed by the laser particle counter, Site 863 . A. Porosity and mean grain size vs. depth. B. Porosity and sorting vs. depth. C. Sorting vs. mean grain size. D. Sorting and mean grain size vs. skewness. See Figure 3 for explanation of symbols.

currents (Heezen and Hollister, 1963; Hollister and Heezen, 1972; Bouma and Hollister, 1973; Stow and Lovell, 1979; McCave et al., 1981). Contour currents may combine with turbidity flows to form nepheloid layers (Eittreim et al., 1975; McCave and Swift, 1976; Pierce, 1976; Swift, 1976).

The transition from sediment slumps to debris flows and turbidity flows likely produce a continuum of deposits going downslope (Hampton, 1972; Leeder, 1982). In general, the thickness and grain size of these deposits decrease in the distal direction. The suite of samples analyzed here, from Sites 861,860 , and 859 , represent the progressively more distal facies of Figure 8. The debris flows are more abundant in the proximal regions and are composed of matrix-supported gravels (not part of the physical properties sample set). Presumably some debris flows would travel farther downslope than others, resulting in interbedded debris-flow and turbidity-flow deposits. Also, both of these types of deposits may well be interbedded with the background sediments composed of hemipelagic organics and suspension fallout from meltwater plumes.

The deposition of silts and clays from meltwater plumes are probably a significant source of material in the slope environment for Leg 141. The plumes may be seasonal in character with periods of higher meltwater discharge supplying greater volumes of mud, especially when the ice extended out onto the continental shelf. The finer components of the mud travel farther and dominate the suspension fallout 
A

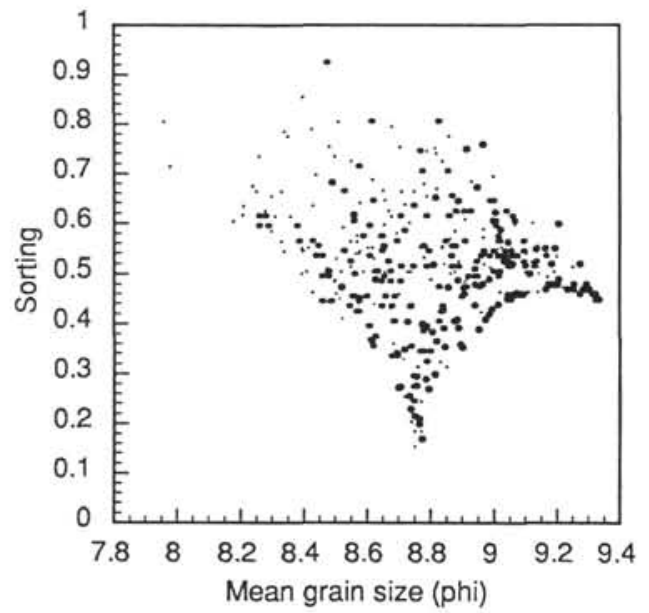

B

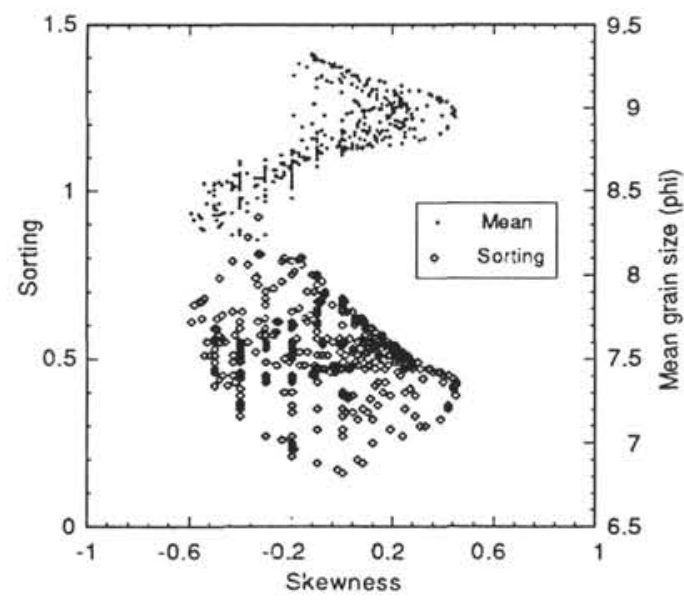

Figure 7. Composite textural data for non-sandy samples analyzed by the laser particle counter, Sites $859,860,861$, and 863 . A. Sorting vs. mean grain size. B. Sorting and mean grain size vs. skewness. See Figure 3 for explanation of symbols.

in deeper water settings. Finer components of the fallout muds may also dominate in proximal settings during intervals of low discharge (e.g., winter in a glaciated source area). Distinguishing suspension fallout and turbidity-flow muds in distal settings is problematical. In proximal settings, suspension fallout muds may be recognized as rhythmites of mud interbedded with sandy turbidites and/or matrixsupported gravelly debris-flow deposits.

The deposition of hemipelagic organic material is expected in zones of coastal upwelling. Primary production of radiolarians, diatoms, foraminifers, and coccolithophores at nutrient-rich upwellings could supply seasonally abundant amounts of organic detritus. The presence of such detritus is best detected in regions with low clastic sedimentation rates. Because the Leg 141 sites are adjacent to a tectonically active glaciated forearc and have high clastic sedimentation rates, the biologic component of the suspension fallout is volumetrically small. However, times of prolific organic activity could produce a significant amount of organic material with a restricted range of test sizes. The presence of a biologic component can be tested for by SEM analysis.

Contour currents are also capable of transporting sediment in the slope environment. These currents can be as strong as $0.5 \mathrm{~m} / \mathrm{s}$ and may be locally erosive (Leeder, 1982). Contour currents, and the muddy portions of turbidity flows, are probable sources of material for nepheloid layers (zones of high suspended sediment near the seafloor). Contourites tend to be rhythmically bedded, well sorted, and lack grading in contrast to poorly sorted and graded, thin distal turbidites (Leeder, 1982; Stow and Lovell, 1979; McCave et al., 1981).

Figures $3,4,5,6$, and 7 illustrate distinctive patterns in the grain size versus sorting plots. The very well sorted samples at grain sizes of 8.6 to 8.8 phi in Figures 3C, 4C, 5C, and 6C may be the result of contour currents reworking the bottom sediments to produce very well sorted, ungraded deposits. Alternatively, the well sorted material may be test material from hemipelagic organic activity. Although the origin of this 8.6 to 8.8 phi, well sorted facies (Facies 1) remains unclear, it probably represents one type of sedimentary process acting in the slope environment explored during Leg 141. Sorting worsens as grain size decreases at Site 859 (i.e., the data defines a limb that rises to the right in Fig. 3C). The upper right end of the limb in Figure $3 \mathrm{C}$ could be a poorly sorted fine-grained facies (Facies 2 ) produced by a second sedimentary process: suspension fallout in a distal setting. Furthermore, this fine-grained ( $>8.8$ phi), poorly sorted Facies 2 seen at all of the sites may represent discrete intervals during which clay-rich suspension fallout occurred. Its occurrence could represent climatic oscillations and/or the absence of bottom currents that would otherwise prevent the deposition of Facies 2. It is possible that the intermediate values along the limb represents a mixing of Facies $I$ and
2. If this interpretation has merit, the Site 859 deposits could be explained by the interaction of two sedimentary processes.

The other sites (Sites 860,861 , and 863) have rhomboid patterns defined by limbs rising to the right (possible origin discussed above) and limbs rising to the left from the very well sorted 8.7 phi Facies 1 samples. The limb rising to the left and the scatter comprising the central area of the rhomboids comprise Facies 3 and may represent the contribution of turbidity flows. Presumably, turbidity flows would contain a range of sizes even in muddy deposits and the graded bedding would result in a worsening of sorting for all mean grain sizes. As grain size increases, as a result of higher energy flows, the degree of sorting would worsen to result in the limb of the rhomboid that rises to the left. Mixing of Facies 1 and 3 could account for the limb climbing to the left on the margin of the rhomboid pattern, as bottom scouring associated with turbidity flows could resuspend and mix with previously deposited contourites.

\section{Grain-size Distributions and Physical and Chemical Properties}

One of the key parameters in understanding the physical and chemical characteristics of marine sediment is the grain size and packing of the sediment (Meade, 1964; Hamilton, 1971, 1974; Hein, 1989). By combining knowledge of texture with mineralogy, loading, and pore-fluid chemistry, models for the response of sediment to burial can be constructed. Furthermore, such physical properties as void ratio, permeability, porosity, specific gravity, shear and compressive strength, and elastic and plastic parameters can be estimated. Also, it may be possible to reconstruct the histories of stress and pore-fluid conditions during diagenesis if sufficient data on the texture, composition, and physical and chemical properties of the sediment have been obtained.

Porosity values for representative lithologies were systematically determined during Leg 141 (Behrmann, Lewis, Musgrave, et al., 1992). Portions of this data are summarized in Figure $2 \mathrm{~A}$ (for the fine-grained sediments lacking pervasive carbonate cements). Three significant trends emerge when porosity data are plotted with respect to grain size: (1) these sediments generally show greater loss of porosity for a given depth than for many other clayey sections reported in the literature (Fig. 2B), (2) there are systematic differences in porosity loss between holes, and (3) there are variations (or deviations from a generalized porosity-loss curve) in the individual downhole data sets. These three observations are discussed below.

First, the compaction of the sediments (porosity in all the holes is reduced to $<30 \%$ ) is substantially greater than that reported for similar 


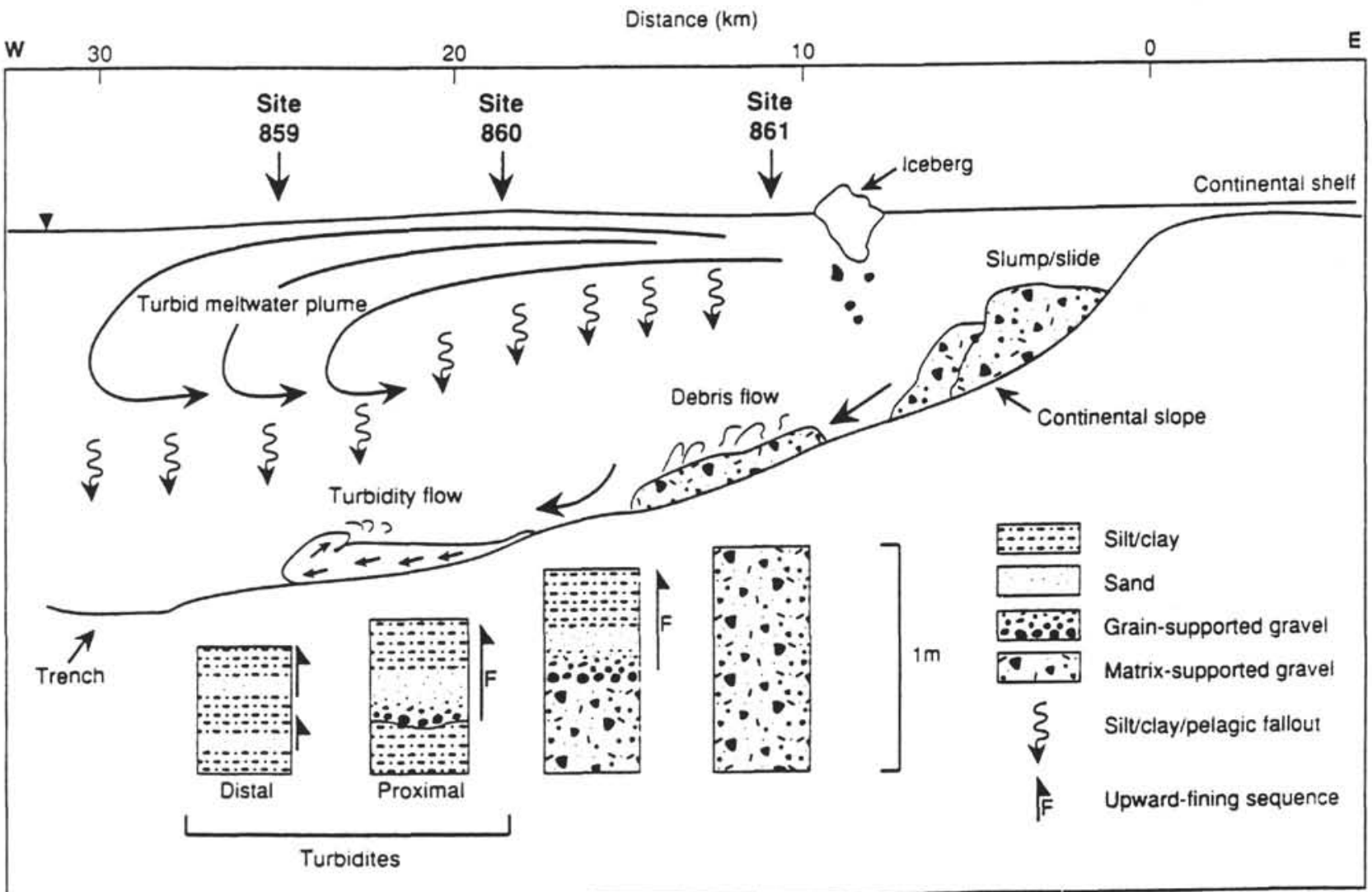

Figure 8. Depositional facies model for the continental slope in the Chile Margin Triple Junction region. The model illustrates many of sedimentary processes acting in the slope environment including debris flows, turbidity flows, and suspension fallout from meltwater plumes and biogenic activity. The model also depicts many of the deposits present at Leg 141 Sites 859,860 , and 861, including turbidites, debris flow, and suspension fallout deposits.

depths from most other basinal environments (Athy, 1930; Hedberg, 1936), as well as laboratory compaction tests (Skempton, 1953, 1970). Comparison of the mean grain sizes observed in Leg 141 samples does not, however, reveal any quantitative difference that could separate these clayey sediments from others in the literature based on mean size alone. This conclusion does not, however, prevent other grain-size distribution parameters having an effect on porosity. As seen in the plots of sorting versus mean grain size, and skewness versus mean grain size, each site on the Chile margin has distinctive grain-size distribution parameters. Therefore, it is feasible that grain-size distributions have affected the compaction history at each site by controlling porefluid migration and the evolution of effective stresses during burial.

Alternatively, the lower-than-expected porosities of the sediments at these sites may be due to one of three compositional attributes: (1) all sites have experienced a common diagenetic history involving advanced potassium fixation or monovalent cation exchange in mixed-layer smectites (or transformations from montmorillonite to metamontmorillonite) (Meade, 1964; Rieke and Chilingarian, 1974), (2) the sediments have a greater abundance of clay mineral fabrics lacking colloidal character (Skempton, 1970), or (3) the glacio-fluvial erosion of the Patagonian Andes has resulted in clay-size fractions that may not be clay minerals (see discussion of shipboard physical properties data; Behrmann, Lewis, Musgrave, et al., 1992). Quantitative mineralogic analyses are not available to evaluate the latter two ideas. From a diagenetic viewpoint, it is clear that the margin has experienced unusual thermal and chemical pore-fluid conditions (Behrmann, Lewis, Musgrave, et al., 1992; also see Sawyer et al., Brown et al., and Froelich et al., all this volume). Most notable is the geochemical evidence for a potassium sink below $400 \mathrm{mbsf}$ at Site 863 that corresponds with observed reductions in the smectite/illite ratio among the shipboard semiquantitative XRD data.
Whatever the causes for the porosity-loss trends, the systematic differences in porosity-loss trends observed between the Leg 141 sites need explanation. The porosity-loss curves show a systematic shift to higher porosity values (for a given depth) moving up slope from Site 859 to Site 860 and then to Site 861 . The skewness versus grain size plots (Figs. 3D, 4D, and 5D) show that the samples from the three sites have a greater abundance of coarser and more negatively skewed grain-size distributions moving upslope from Site 859 to Site 860 , then Site 861 . The sorting versus mean grain-size plots (Figs. 3C, 4C, and 5C) indicate that sorting also diminishes upslope. Could the differences in the porosity curves be a simple function of these textural differences? This does not appear to be the most likely alternative for a number of reasons. First, both theoretical and empirical studies of compaction (Hamilton, 1974; Rieke and Chilingarian, 1974: Skempton, 1953, 1970) argue that the better sorted units that dominate low on the slope at Site 859 should theoretically lead to higher, not lower porosities as observed at this site. The same studies also argue that coarser mean grain sizes found upslope at the three sites should correlate with progressively lower porosities rather than the observed trend toward higher porosities at these sites. Second, the other lower slope site, Site 863, which has overlapping grain-size characteristics with Site 861 , also shows "accelerated" porosity loss with depth (i.e., similar to Site 859). Thus, the observed systematic differences observed in porosity between the holes do not appear to behave as predicted by theoretical and empirical work for sediments with textures similar to those measured in this study. In general, the systematic differences seen between the sites appear to correlate more with position on the slope than with the textural parameters measured in this study. For example, Sites 859 and 863 , both located at the toe of the slope, show the greatest porosity losses with depth even though 
Site 859 has the finest grain-size material and Site 863 has the coarsest material (comparable to Site 861).

There are three alternative hypotheses that may explain the location-dependent systematic differences between Leg 141 sites: (1) post-compaction differential uplift (and erosion) at Sites 859 and 863 , (2) site-specific thermal and chemical conditions that have affected the pore fluids and clay minerals during diagenesis differently at each site, and (3) substantially different primary mineralogy at Sites 859 and 863 . The data presented by Kurnosov et al. (this volume) address the mineralogic differences among these sites. The papers by Brown et al. and Froelich et al. (both this volume) address thermal and chemical diagenetic conditions.

The high frequency shifts seen within the porosity-loss trends downhole for a given site are another type of variation observed within the porosity data. High-frequency shifts in porosity with depth below seafloor exist at all the sites, but they do not appear to have common causes between sites. For example, higher porosity is associated with coarser grain size at Site 859 but finer grain size at Site 860 . Higher porosity is associated with better sorting at Site 859 but is not affected by sorting at Site 860 . Reduced porosity is associated with better sorting at Site 861 and coarser grain size at Sites 861 and 863. Despite the lack of common causes between sites, high-frequency changes in porosity-loss with depth provides useful information for individual sites. The porosity-loss trend that is most striking is seen at Site 859 . At Site 859 there are oscillations at 50-80,360-415, and $>460$ mbsf, where porosities are higher than the general trend. At each of these intervals the degree of sorting and mean grain size increases (Figs. 3A and $3 \mathrm{~B})$. These correlations, taken together with the theoretical work discussed above (Hamilton, 1974; Rieke and Chilingarian, 1974; Skempton, 1953, 1970) suggest sorting, rather than grain size, could be an important control on porosity at Site 859 . The inference is that well-sorted zones form the high-porosity, high-permeability pathways for fluid migration within the section. Noteworthy is the fact that three of four occurrences of bitumen with mature carbon preference indices (CPI) are located in or just above these zones of relatively high porosity, well sorted material (Behrmann, Lewis, Musgrave, et al., 1992).

Between 240 and 260 mbsf at Site 859 the degree of sorting decreases sharply (Fig. 3B), which does not correlate with the porosity data but appears to coincide with three observations for this interval: (1) a thermal "spike" in the downhole temperature data, (2) sharp breaks in the inorganic pore-water chemistry trends, and (3) the highest smectite/illite ratio in the semiquantitative shipboard XRD analyses. The argument has been made (Behrmann, Lewis, Musgrave, et al., 1992) that this interval was a zone of thermal fluid advection with sufficient potential to drive a hydrothermal alteration of illite to smectite. If so, the formation of a diagenetic clay mineral phase could explain the decrease in sorting observed for this interval.

A number of reversals in the downhole ages of the stratigraphy at Site 860 were inferred based on the paleontological data. The combined age, sedimentologic, and structural data were used to support the concept of an imbricated section, with repetitions of section resulting from faulting occurring in the intervals $210-240,300-310$, $420-430,520-530$, and 580-590 mbsf. The suggestion that faults may be repeating the section was difficult to test on sedimentologic grounds because of the lack of distinctive marker layers or identifiable packets of strata. The grain-size analyses for this site show that there are five distinctive oscillations in the ranges of both the mean grain size and sorting with depth below seafloor for these holes (Figs. $4 \mathrm{~A}$ and $4 \mathrm{~B}$ ). If we accept that the sorting versus mean grain-size plots reflect mixing of Facies 1, 2, and 3, the oscillations seen here in the grain-size data support the imbrication model, with an average thickness of the repetition of $100-120 \mathrm{~m}$. Alternatively, these could represent cyclic depositional events, with the periodicity set by climatic controls on the relative contributions of clastic and pelagic suspension fallout to the slope deposits.

At Sites 861 and 863, which contain coarser units (turbidites), there are similar, but not as clearly defined, small- to medium-scale
(20-60 m) oscillations of porosity and grain size with depth below seafloor (Figs. 5A and 6A). In general, where obvious correlations can be made (such as the porosity shift at Site 863 between 400 and $500 \mathrm{mbsf}$, where it decreases by $10 \%$ from about $37 \%$ to $27 \%$; Fig. $6 \mathrm{~A})$, decreases in porosity corresponds with increases in mean size. However, there is no corresponding shift in sorting at this interval (Fig. 6B). Thus, the sharp decrease in porosity between 400 and 500 mbsf for Site 863 may not be texturally controlled. Rather, it may be depth or diagenetically controlled. Recall that Site 861 had grain-size distributions similar to those at Site 863 but maintained porosities close to $40 \%$ at depths where Site 863 has porosities close to $27 \%$ (e.g., 500 mbsf; Figs. 5A and 6A). Thus, as one moves from smallscale (at a site) to large-scale (between sites) variations in physical properties, textural variations seem to have less influence compared to the depositional and tectonic setting of the deposits.

\section{CONCLUSIONS}

Leg 141 of the Ocean Drilling Program recovered predominantly clays and silty clays from four sites drilled into the inner trench wall and slope in the vicinity of the Chile Margin Triple Junction. Quantitative grain-size analysis for a suite of nearly 600 samples obtained directly from the shipboard physical-properties sampling intervals has been completed. Each site exhibits a distinctive pattern of sorting and mean grain-size parameters, suggesting differential mixing of three end-member facies, which may include contourites (or hemipelagic organics) (Facies 1), suspension fallout (Facies 2), and distal turbidites (Facies 3). Comparisons of down-hole variations in the grain-size parameters with the porosity data suggest textural factors have partly controlled porosity loss during burial. The model developed for a tectonically imbricated section at Site 860 is consistent with the cyclic pattern evident in the grain-size data set. Large-scale differences seen between holes in the porosity-loss curves are probably the result of variations in lithologies due to the depositional setting, tectonic setting, and diagenetic history.

\section{REFERENCES}

Athy, L.F., 1930. Density, porosity and compaction of sedimentary rocks. AAPG Bull., 14:1-24.

Behrmann, J.H., Lewis, S.D., Musgrave, R.J., et al., 1992. Proc. ODP, Init. Repts., 141: College Station, TX (Ocean Drilling Program).

Bouma, A.H., and Hollister, C.D., 1973. Deep ocean basin sedimentation. In Turbidites and Deep Water Sedimentation. Soc. Econ. Paleontol. Mineral., Pacific Sect., Short Course, 79-118.

Carver, R.E., 1971. Procedures in Sedimentary Petrology: New York (Wiley). Eittreim, S., Biscaye, P.E., and Amos, A.F., 1975. Benthic nepholoid layers and the Ekman thermal pump. J. Geophys. Res., 80:5061-5067.

Folk, R.L., 1974. Petrology of Sedimentary Rocks: Austin (Hemphill Publ.).

Galehouse, J.S., 1971. Sedimentation analysis. In Carver, R.E. (Ed.), Procedures in Sedimentary Petrology: New York (Wiley), 69-94.

Halka, J.P., Conkwright, R.D., Kerhin, R.T., and Wells, D.V., 1980. The design and calibration of a rapid sediment analyzer and techniques for interfacing to a dedicated computer system. Maryland Geol. Surv., Inf. Circ., 32.

Hamilton, E.L., 1971. Prediction of in-situ acoustic and elastic properties of marine sediments. Geophysics, 36:266-284.

- 1974. Prediction of deep-sea sediment properties: state of the art. In Inderbitzen, A.L. (Ed.), Deep-Sea Sediments: Physical and Mechanical Properties: New York (Plenum), 1-44.

Hampton, M.A., 1972. The role of subaqueous debris flow in generating turbidity currents. J. Sediment. Petrol., 42:775-793.

Hedberg, H.D., 1936. Gravitational compaction of clays and shales. Am. J. Sci, 31:241-287.

Heezen, B.C., and Hollister, C.D., 1963. Evidence of deep sea bottom currents from abyssal sediments. Int. Union Geodetics Geophys., 6:111.

Abbreviations for names of organizations and publications in ODP reference lists follow
the style given in Chemical Abstracts Service Source Index (published by American the style given in $\mathrm{Ch}$.
Chemical Society). 
Hein, F.J., 1989. Slope aprons and slope basins. In Pickering, K., Hiscott, R.N., and Hein, F.J. (Eds.), Deep Marine Environments: Clastic Sedimentation and Tectonics: London (Unwin-Hyman), 91-108.

Hollister, C.D., and Heezen, B.C., 1972. Geological effects of ocean bottom currents: western North Atlantic. In Gordon, A.L. (Ed.), Studies in Physical Oceanography: New York (Gordon and Breach), 37-66.

Leeder, M.R., 1982. Sedimentology: Process and Product: London (Allen and Unwin).

McCave, I.N., Lonsdale, P.F., Hollister, C.D., and Gardner, W.D., 1981. Sediment transport over the Halton and Gardar contourite drifts. J. Sediment. Petrol., 50:1049-1062.

McCave, I.N., and Swift, S.A., 1976. A physical model for the rate of deposition of fine-grained sediments in the deep sea. Geol. Soc. Am. Bull., $87: 541-546$.

McManus, J. 1988. Grain size determination and interpretation. In Tucker, M. (Ed.), Techniques in Sedimentology: Oxford (Blackwell), 63-85.

Meade, R.H., 1964. Removal of water and rearrangement of particles during the compaction of clayey sediments-review. Geol. Surv. Prof. Pap. U.S., 497B.

Pierce, J.W., 1976. Suspended sediment transport at the shelf break and over the outer margin. In Stanley, D.J., and Swift, J.J.P. (Eds.), Marine Sediment Transport and Environmental Management: New York (Wiley), 437-458.
Rieke, H.H., III, and Chilingarian, G.V., 1974. Compaction of Argillaceous Sediments: New York (Elsevier).

Selley, R.C., 1988. Applied Sedimentology: New York (Academic).

Skempton, A.W., 1953. Soil mechanics in relation to geology. Proc. Yorks. Geol. Soc., 29:33-62.

1970. The consolidation of clays by gravitational compaction. $Q$. J. Geol. Soc. London, 125:373-411.

Stanley, D.J., and Swift, J.J.P. (Eds.), Marine Sediment Transport and Environmental Management: New York (Wiley).

Stow, D.A.V., and Lovell, J.P.B., 1979. Contourites: their recognition in modern and ancient sediments. Earth Sci. Rev., 14:251-291.

Swift, D.J.P., 1976. Continental shelf sedimentation. In Stanley, D.J., and Swift, J.J.P. (Eds.), Marine Sediment Transport and Environmental Management: New York (Wiley), 311-350.

Date of initial receipt: 29 July 1993

Date of acceptance: 28 February 1994

Ms 141SR-001 